\title{
EMOTIONAL EMPATHY MODEL FOR ROBOT PARTNERS USING RECURRENT SPIKING NEURAL NETWORK MODEL WITH HEBBIAN-LMS LEARNING
}

\author{
Jinseok Woo' ${ }^{1}$ János Botzheim ${ }^{2}$, Naoyuki Kubota ${ }^{3}$ \\ 1,2,3 Graduate School of System Design, Tokyo Metropolitan University, \\ 6-6 Asahigaoka, Hino, Tokyo \\ E-mail: woojs@tmu.ac.jp ${ }^{1}$, dr.janos.botzheim@ieee.org², kubota@tmu.ac.jp ${ }^{3}$
}

\begin{abstract}
This paper discusses the development of an emotion model for robot partner system. In our previous studies, we have focused only on the robot's emotional state. However, the emotional state of the other party is also an important factor for smooth conversation in human society. Therefore, the robot partner has two emotional structures for human: empathy and robot emotion. First, human empathy uses a perceptual based emotion model to know the human's emotional state based on the sensory information. Next, we propose a recurrent simple spike response model to improve the robot's emotional model, and we apply "Hebbian-LMS" learning to modify the weights in the spiking neural network. The robot's emotional state is calculated by using the human's emotional information, internal and external information. The robot partner can use the emotional results to control the facial and gesture expression. The utterance style is also changed by the robot's emotional state. As a result, the robot partner can interact emotionally and naturally with human. First, we explain the related works and the development of the robot partner "iPhonoid-C". Next, we define the architecture of the emotional model to realize emotional empathy towards human. Then, we discuss the algorithms and the methods for developing the emotional model. Finally, we show experimental results of the proposed method, and discuss the effectiveness of the proposed structure.
\end{abstract}

Keywords: Emotional Empathy Model, Recurrent Spiking Neural Network, Hebbian-LMS Learning, Robot Partner, Conversation System

\subsection{INTRODUCTION}

In recent days, "social" is an important keyword for human life. "Man is by nature a social animal", said Aristotle in 328 BC. It means that human cannot be separated from being social. The meaning of "social" is defined in "Cambridge Dictionaries Online" as "relating to the things you do with other people for enjoyment when you are not working" [9]. Therefore, people put a lot of effort in social relationships. Communication is a core element to human for improving social relationships, and communication skills and emotional statements are important factors to enrich human life. Furthermore, human emotion plays an important role in communication. Thus, we consider communication system and emotional model to realize the socially interactive robots.

Human emotional state change has a deep relations with subjective and intuitive situations [40, 41, 51]. We define subjective situation as the personal representation of an individual, based on his or her own mental model (molded by their experience). Whereas for intuitive situation, it is a group representation where individuals within the group should uphold the same belief. These human emotion theories are discussed by experts [17, 34]. In psychological studies, there are various emotional definitions of human. For example, Plutchik have mentioned about "If there is little consensus on the meaning of the term, it is no wonder that there is much disagreement among contemporary theoreticians concerning the best way to conceptualize emotion and interpret its role in life." [31]. Thus, we could know that many conceptual emotional models exist. Therefore, the aim of this paper is to propose the concept of the robot's emotional model specifically to emulate humanrobot interaction, instead of specifying the real definition of human emotion, because the importance of emotions is significant in human interaction. We have been developing an emotion model based on multilateral research by using engineering viewpoints [26, 27]. 
Generally, human communication includes empathy of emotion [38]. Therefore, the human interaction style changes depending on the emotional state. For example, when another person has a "sad" emotional state, we should consider that factor when communicating with her or him. This idea is similar to the mutual cognitive environment in relevance theory [37]. Accordingly, some researchers have tried to know the human emotional state based on the speech [30]. We have also used adjective information in human utterance sentence that is a direct expression of human emotional state as the input information in the emotion model of the robot [46].

The robot's emotional model can improve the human-robot interaction in order to realize a socially acceptable robot partner. Thus, many researchers have shown interest in developing an emotion model for the robot partner. For example, Kismet can interact with human by facial expression [7, 8]. This is very helpful for smooth interaction in the human society. Another researcher has explained the significance of emotional factors to associative memory for human-robot interaction [28]. In Japan, "Kansei Engineering" has a long history [29]. It is a way to incorporate human sensibility in the manufacturing process. "SoftBank Robotics" developed the robot "Pepper" which has an emotional model [36] and researchers use this robot to develop educational applications with children [39]. In this way, the emotional factor has been applied aggressively from various points of view on human emotions. Speech based emotion recognition systems enable users to determine the speaker's emotional states by extracting corresponding information that resides in speech signals [35].

In [46], we discussed perception based emotion model in order to use conversation system. The robot partner calculates its emotional state based on the utterance sentence of the human. Then, the robot partner can control its utterance pattern, facial and gestural expression based on the emotional parameters.

In [5], we discussed an emotional model based on spiking neural network having a feed-forward structure with no recurrence. Not all objects in view are processed instantaneously in human recognition system, unless a certain threshold such as object saliency is reached. This characteristic can be directly modeled by spiking neural network, which resembles closely the biological mechanism of neurons. Thus we used spiking neural network structure for the emotional model mechanism. Hebbian learning was applied to modify the neural network's weights [16]. However, the model has some limitations. One big problem with this approach was that the weights could only increase until they reached saturation. In order to prevent saturation, in this paper, we use Hebbian-LMS learning method.

We also considered the design of emotion based on recurrent structure in the feeling layer, and we use feedback from the mood layer to the feeling layer. Our emotion model has time scales for sustainable emotions. The feeling layer that directs expression of the emotion has a short-term time scale. This feeling easily changes the emotion state according to the external and internal stimulus. The mood layer has a long-term time scale. Mood has a long time range influence, longer than the feeling layer has. Some time, feeling state is affected from mood state. Thus, we use

mood information in the feeling state.

Normally, the current emotional information plays more important role than the previous emotional information in the memories [42]. However, by taking into account the previous information stored in the memory, it can realize a finer model of emotion that better models the human emotion. It is possible for recurrent network to make short or long term memory, but feed forward network cannot have a memory. This is because feed forward structure uses only current information. In our recurrent model, we can use the feedback from the mood to the feeling layer, and we can also use the previous feeling values in the feeling layer. This fact is very important when realizing the emotional state mimicking human emotion.

Thus, we modify the previous feed-forward only structure with no recurrence to a recurrent model that has feedback. We propose a recurrent structure to refer to the previous feelings when updating the current feelings. We introduce these emotional model structures in our robot partner. We use two emotional models, a perception based emotional model and a recurrent simple spike response model based emotional model.

In the perception based emotion model, the state of human subjective emotion is applied to the robot. The perception based emotional model calculates the human emotional state by using adjective, gesture, and smile information. One important advantage of our model is that the robot can build its own emotional model by unsupervised learning using the Hebbian-LMS algorithm. In this recurrent simple spike response model based 
emotional model, the robot's emotional state changes depending on the human feeling state result, battery, distance, and gender information. The human emotional state has a relation to the previous and current feeling states. Accordingly, we can consider, that the robot partner should have a similar structure of emotion. Furthermore, we also consider the feedback from mood layer to feeling layer. Thus, the feeling state can depend also on the mood state.

This paper is organized as follows. Section 2 presents the proposed robot partner system. Section 3 explains the conversation system for the robot partner. Section 4 introduces the proposed emotional model of the robot partner. Section 5 presents experimental results. Conclusion and future work are presented in Section 6.

\subsection{SMART DEVICE BASED ROBOT PARTNER SYSTEM: iPhonoid-C}

We have developed "iPhonoid-C" as a robot partner to interact with human in [45]. The robot partner consists of two parts: a smart device and a body (Fig. 1). The smart device part is in charge of computational intelligence and thereby control the hardware and the body $[46,48]$. In this paper, we focus on emotional empathy model by recurrent simple spike response model better than the development of robot hardware system.

The emotional state is a very important factor in human communication [32]. For example, we should consider other people's emotional state to avoid hurt feelings when we talk, because the meaning of human utterance can vary depending on the emotional state of human. This emotional model will be explained in Section 4.

The affecting factors of emotional state change can be classified as external and internal stimulus [24]. External emotional factors refer to "social" and "non-social" factors. On the other hand, internal emotional factor is "physiological state to complex cognitive activities". Consequently, sensory information are required in order to use the emotional model. In this paper, sensors in the smart device are used similarly as human sense organs in order to model the robot's emotion. iOS device has various sensors e.g., display touch status, camera, mic, compass, battery status, accelerometer, proximity status, and shake information [1, 2, 3]. The detailed information is shown in Table 1. For the experiment, we define that all sensory signals scale from 0 to 1 as an output value (Table 2). The sensory information is updated in every 0.5 second.

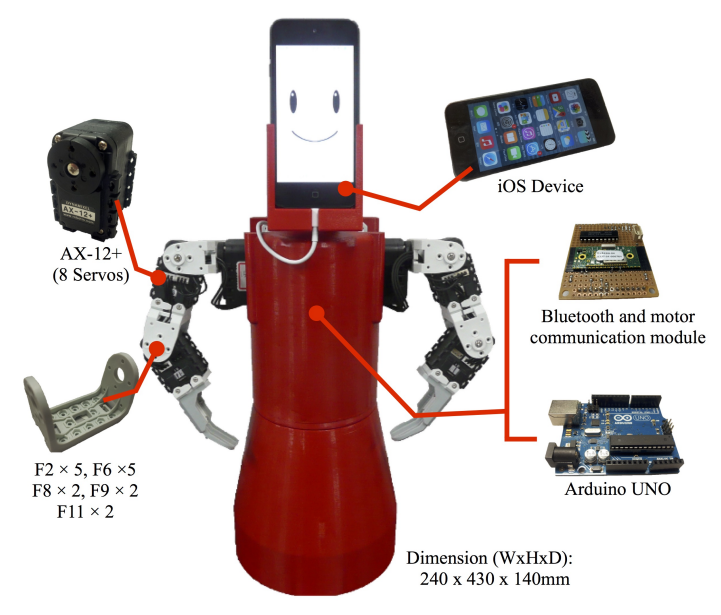

Fig. 1: Robot partner: iPhonoid-C 
Table 1: Type of sensor information from smart device

\begin{tabular}{|l|l|}
\hline Sensor & Information \\
\hline \hline Camera & RGB information \\
\hline Microphone & Sound level information \\
\hline Touch interface & Touch and swipe information \\
\hline GPS & Geometric information \\
\hline Proximity & $\begin{array}{l}\text { Presence of nearby object } \\
\text { information }\end{array}$ \\
\hline Compass & Geometric direction \\
\hline Accelerometer & Accelerometer information \\
\hline Gyroscope & Movement information \\
\hline Battery status & Battery charging level \\
\hline
\end{tabular}

Table 2: Sensory information from smart device

\begin{tabular}{|l|l|c|}
\hline Input information & Parameters (Sampling interval: Every 0.5 second) & Output value \\
\hline \hline Human gesture & Hand up and down, left and right & 0 or 1 \\
\hline Object color & Human skin color, red, blue & 0 or 1 \\
\hline Display touch & $\begin{array}{l}\text { Gesture actions: } \\
\text { Tap, long press, swipe about 4 direction (up, down, } \\
\text { left, right) }\end{array}$ & 0 or 1 \\
\hline Touched finger radius & $\begin{array}{l}\text { The contact area of the finger: } \\
\text { 20mm (min) - 100mm (max) }\end{array}$ & $0.0-1.0$ \\
\hline Human detection & Human or nobody & 0 or 1 \\
\hline Human distance & $\begin{array}{l}\text { Distance between human and robot: } \\
1.5 m \text { (max) }-0.1 \mathrm{~m} \text { (min) }\end{array}$ & $0.0-1.0$ \\
\hline Smile & Information of smiling or not & 0 or 1 \\
\hline Gender & Information of female or male & 0 or 1 \\
\hline Racial & Information of asian or non-asian & 0 or 1 \\
\hline Proximity sensor & Nothing or somthing & 0 or 1 \\
\hline Mic volume & Volume level: $-120($ min), $0($ max) & $0.0-1.0$ \\
\hline Body shake & Detecting shake event or not & 0 or 1 \\
\hline Compass direction & Parameter changing from North to south & $0.0-1.0$ \\
\hline Battery status & $\begin{array}{l}\text { Battery charging level: } \\
\text { 0 (Low power), 1 (full charged) }\end{array}$ & $0.0-1.0$ \\
\hline
\end{tabular}

\subsection{COMMUNICATION SYSTEM}

The robot's communication system consists of two parts: verbal communication (Section 3.1) and nonverbal communication (Section 3.2).

\subsection{Verbal Communication}

In [46], we have proposed a conversation module as shown in Fig. 2. This proposed conversation system has three main components to interact with human. The first component is the Conversation Flow Utterance System (CFUS); the second component is the Sentence Building Utterance System (SBUS), and the third component is the Time Dependent Utterance System (TDUS). Fig. 2 illustrates the control of the conversation flow between the three main components. When user schedule information is available, then TDUS part is applied. If not, then 
SBUS is used. If the human utterance sentence is declarative, then CFUS including verb and adjective comparison is applied based on the type of the sentence.

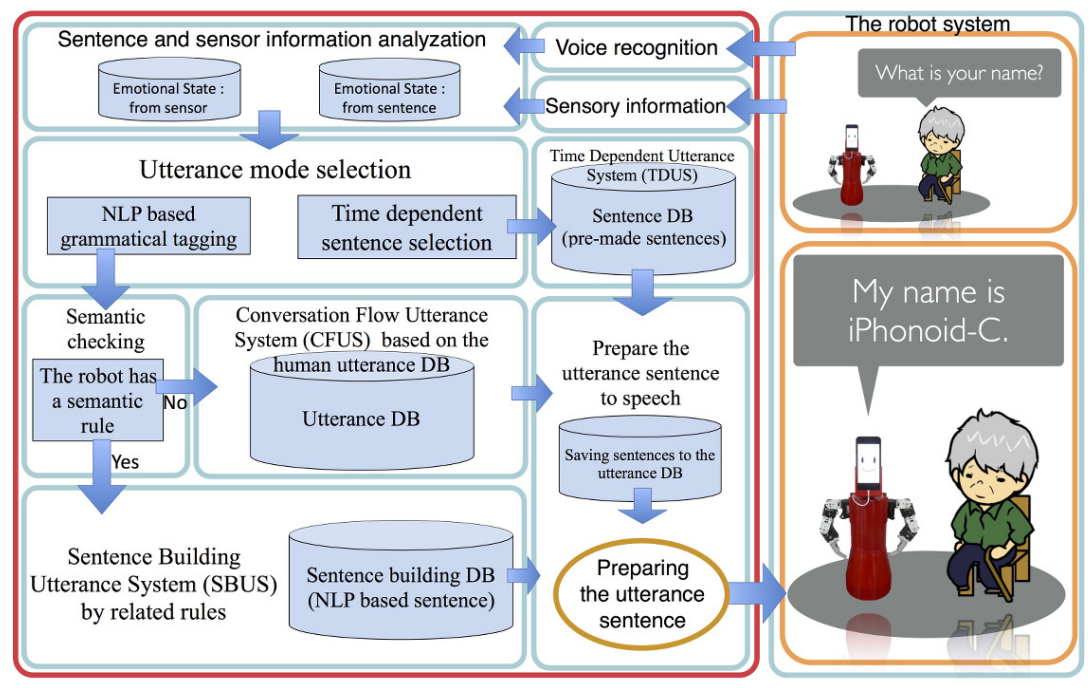

Fig. 2: The structure of the conversation system

\subsection{Nonverbal Communication}

In [45], we have proposed facial and gestural expression by using emotional state in nonverbal communication. Facial expression uses a simple fuzzy model to change the robot's face based on its emotional state.

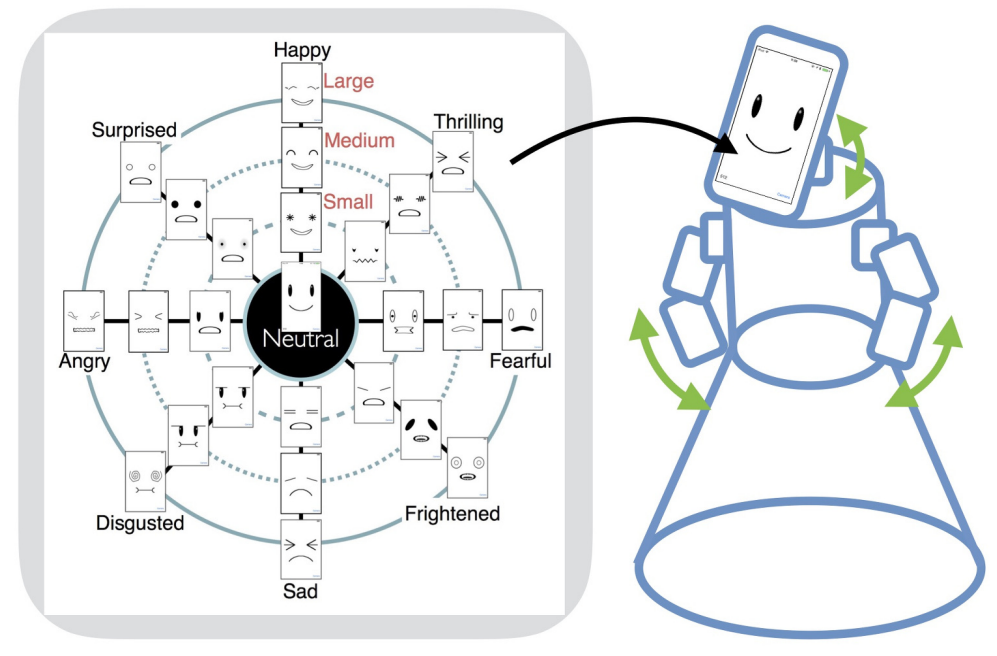

Fig. 3: Emotional expression by facial and gesture expression

The facial expression has three levels based on the mood information using two types of mood parameters to change the robot's face: positive and negative moods. We have also proposed gestural expression based on the emotional state using Laban Movement Analysis (LMA) [45]. This system uses the action drive based on the three effort factors: Space, Weight, Time. The gestural expression part uses the feeling parameter to create a 
chromosome structure for motor control. The results of emotions are used for robot facial and gesture expression [45] and robot conversation [46] (Fig. 3). For example, when robot feeling is "happy" and mood difference between positive and negative mood is "big", the robot partner will use large happy facial and gestural expression.

\subsection{EMOTIONAL MODEL TO HUMAN ROBOT INTERACTION}

Human emotion plays an important role both in social communication and in human decisions (e.g., decision of avoiding dangerous situation) $[4,25]$. Emotional state is a very important factor to generate facial and gestural expressions. These expressions are dynamically changing based on the emotional states [12, 13, 19].

The aim of our robot development is to realize a social robot, which can be achieved by developing emotional models in the robot partner [44, 45, 49]. Various attempts have dealt with human emotional state using a computational model, such as the analysis of emotion based on the facial expression [18] and emotional model based on computational neural network modeling [22].

In [49], we discussed perception based emotional models. In our previous studies [46, 47], we have focused only on the robot's emotional state. However, the emotional state of the human is also an important factor for smooth conversation in human society [11]. Therefore, we defined human as having two different emotional parts for interaction. In Fig. 4, each human has "Own" and "Other" emotional structure. The following explanation is described from the robot's point of view.

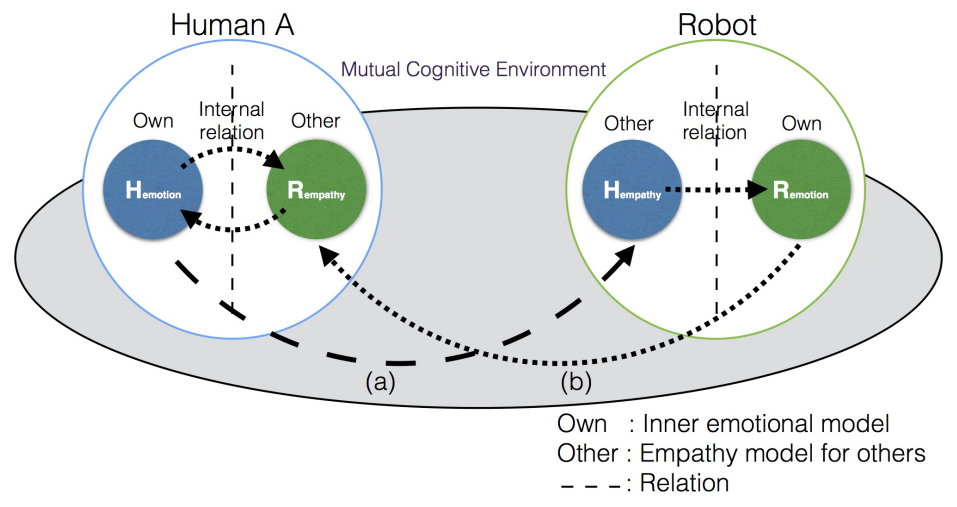

Fig. 4: The structure of emotional relationship between human and robot

"Other" structure is emotional empathy from others (in our explanation, the human) as shown in figure 4 (a) (e.g., The robot feels the human is angry). This emotional state is inferred from human state by using perceptual information to indirectly obtain the human emotional state (Figure 5). In order to know the meaning of human actions and utterances indirectly, the robot should consider human behavior. However, it is impossible to determine the state of human real emotional state. Thus, human emotions are inferred depending on the human's reaction as a response to the human-robot interaction (e.g., human facial and gestural expression, distance between human and robot). Therefore, other human's emotional states are estimated based on the sensory information. For example, when the human is getting away from robot, robot can consider this situation to infer human emotional state. Therefore, we consider this model to use emotional empathy structure. This will be presented in Section 4.2.

For perceptional information, we should consider sensory information as well (Section 2).

On the other hand, "Own" structure is the robot's inner emotional model (e.g., I am happy). The own emotional state is calculated by using human's emotional information, internal state and external input for robot partner. That is, the robot partner's emotional state is changed not only by environmental information but also by 
human's emotional states. This information is used to determine the robot's feeling and mood state. By this structure of emotional empathy, robot can get the basic information of "empathy" after recognizing the emotional results. For the robot's emotional model, we present our new model using the recurrent simple spike response model with Hebbian-LMS learning. This will be introduced in Section 4.3. Therefore, we integrate two emotional structures in order to use emotional system on the robot partner (Fig. 5).

In this paper, we apply "Hebbian-LMS" learning method to update the robot own emotional weight parameter to form the robot emotional state. Saturation problem is also considered. "Hebbian-LMS" learning method performs unsupervised learning [43]. Therefore, the robot partner is able to build its emotional model by itself.

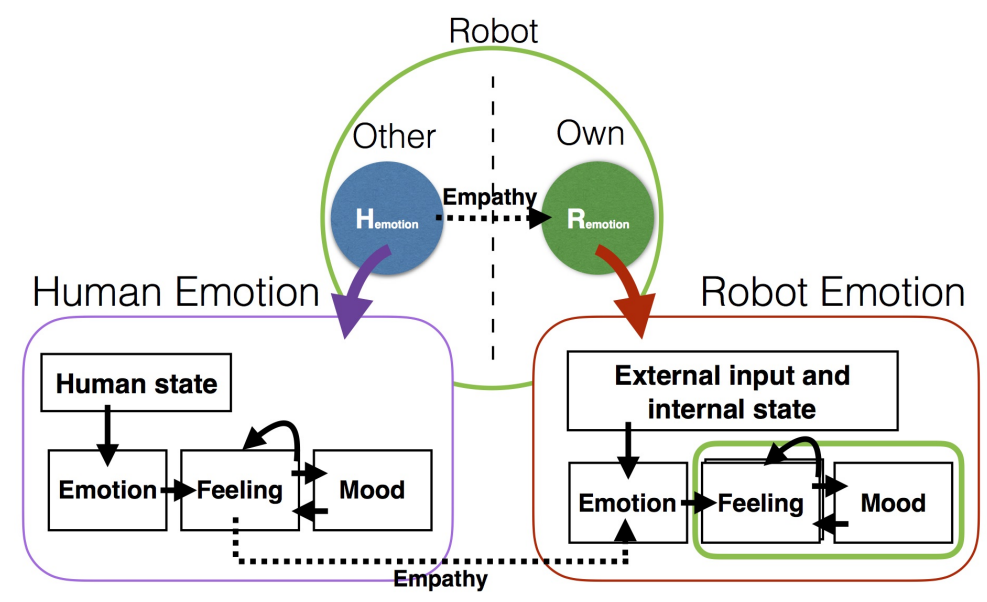

Fig. 5: The information flow of emotional empathy model

\subsection{Emotional Parameters for Robot Emotion}

There are several emotional factors and human emotion that do not have a commonly accepted definition. Hence, many parameters of emotion can be considered in the robot's emotional model. We use six pieces of predefined sensory information in the emotional parameters as follows:

1. Human gesture information: Human gestures are related to the components of emotion [19]. In this paper, two human gesture states are used in the emotional model. The robot interprets predefined meaning of the gestures. Up and down gestures have positive meaning while left and right gestures have negative meaning. Basically, the robot is interested in positive response.

2.Human smiling state: Human facial expression is a good factor to know the emotional state. If human is smiling, then the robot considers that the person is in a happy state.

3.The adjective contained in human words: Human utterance sentences include emotional information. Therefore, researchers considered to analyze the emotional state by sentence [21, 50]. We utilize the adjective information from the human utterance sentences in order to infer the human emotional state. The adjective information based on our paper in [46] is presented in Table 3. If the sentence contains negation (such as the word "not") then the parameters change from $(x)$ to $(1-x)$. For example, when the adjective is "Happy", the parameter related to surprise is " 0.8 " in Table 4 . However, if the adjective contains a negation like "not happy", the surprise parameter is used with " 0.2 ". The " 0 " parameter is not considered to change in Table 4.

4.Robot battery state: Human emotional state can also change based on the internal information in the body. Therefore, we use the battery information of the smart device to mimic human hunger state. The fully charged state means that the robot is happy. 
5.Distance from human: The robot was given the habit to feel better when it is closer to human. Hence, the robot partner has happy emotional state when the human is closer, and the robot partner has sad emotion when the human leaves the robot.

6.Human gender: The robot has different emotional parameters describing its personality. We considered that emotional state varies based on the gender difference [10, 14]. Thus, in this paper, the information of gender was taken into consideration as the difference between the parameter of emotion model [20]. Here, as the basic properties, the robot was given a more positive emotional state when the parameter is "female".

Table 3: Adjective words related to human emotional parameter

\begin{tabular}{|l|l|}
\hline Feelings & \multicolumn{1}{c|}{ Adjective words } \\
\hline Neutral & - \\
\hline Happy & nice, glad, great, good, pleased \\
\hline Surprise & wonder, awe, amazing, curiosity, astonished \\
\hline Angry & enraged, heated, furious, uptight, bad \\
\hline Disgusting & nasty, hateful, fulsome, shocking, awful \\
\hline Sad & heartbroken, sorry, wistful, bitter, sick \\
\hline Frightened & anxious, panicky, spooked, startled, unnerved \\
\hline Fearful & afraid, hesitant, nervous, scared, tense \\
\hline Thrilling & frantic, sensational, rousing, miraculous, breathtaking \\
\hline
\end{tabular}

Table 4: Coefficients between emotions and feelings

\begin{tabular}{|l|c|c|c|c|c|c|c|c|c|}
\hline Adejctive words & Neutral & Happy & Surprise & Angry & Disgust & Sad & Frightened & Fearful & Thrilling \\
\hline Neutral & 0.0 & 0.1 & 0.0 & 0.0 & 0.0 & 0.0 & 0.0 & 0.0 & 0.0 \\
\hline Happy & 0.0 & 1.0 & 0.8 & 0.0 & 0.0 & 0.1 & 0.1 & 0.0 & 0.7 \\
\hline Nice & 0.0 & 0.9 & 0.7 & 0.0 & 0.0 & 0.0 & 0.1 & 0.0 & 0.6 \\
\hline Glad & 0.0 & 0.9 & 0.6 & 0.0 & 0.0 & 0.0 & 0.1 & 0.0 & 0.5 \\
\hline Great & 0.0 & 0.9 & 0.5 & 0.0 & 0.0 & 0.0 & 0.1 & 0.0 & 0.7 \\
\hline$\ldots$ & $\ldots$ & $\ldots$ & $\ldots$ & $\ldots$ & $\ldots$ & $\ldots$ & $\ldots$ & $\ldots$ & $\ldots$ \\
\hline Breathtaking & 0.0 & 0.4 & 0.3 & 0.1 & 0.1 & 0.1 & 0.1 & 0.5 & 0.0 \\
\hline
\end{tabular}

\subsection{Emotional empathy from estimated human emotion}

The emotional input $u_{b, c, d}^{\mathrm{E}}(t)$ is generated based on the adjective word information in the human sentence as follows:

$$
u_{b, c, d}^{E}(t)= \begin{cases}1 & \text { if }\left(S_{\text {adjective }}=S_{b}\right) \wedge\left(G_{\text {gesture }}=G_{c}\right) \wedge\left(D_{\text {smile }}=D_{d}\right) \\ 0 & \text { otherwise }\end{cases}
$$

The $(b, c, d)$ are item numbers. $S_{\text {adjective }}$ is the adjective in the human sentence and $\mathrm{S}_{b}$ is the adjective group related to the $i$-th emotion as presented in [46]. For example, $S_{l}(b=1)$ is adjective word number 1 (e.g., $S_{l}=$ "happy"). The adjective words are classified into eight groups, one group for each feeling, and six adjective words are included in each group. Each adjective word has an ID number used for calculating the emotion parameter. $G_{\text {gesture }}$ is the human gestural information. $G_{c}$ is related to the $i$-th emotion, either moving hand up and down vertically $\left(G_{0}\right)$ or moving hand left and right horizontally $\left(G_{1}\right)$. $D_{\text {smile }}$ is the human facial information of smile. $D_{d}$ is related to the $i$-th emotion, where $D_{l}$ means smiling, $D_{0}$ means no facial expression.

The state of the $i$-th feeling $u^{\mathrm{F}}{ }_{i}(t)$ is updated by the emotional input from the viewpoint of bottom-up construction and the top-down constraints from mood values are also considered: 


$$
u_{i}^{F}(t)=\tanh \left(\kappa u_{i}^{F}(t-1)+(1-\kappa)\left[E_{i}+F_{i}\right]\right)
$$

where

$$
\begin{aligned}
E_{i} & =\sum_{b, c, d=0}^{N^{E}} w_{i, b, c, d}^{E} \cdot u_{b, c, d}^{E}(t-1) \\
F_{i} & =\sum_{j=0, j \neq i}^{N^{F}} w_{i, j}^{F} \cdot u_{j}^{F}(t-1) \\
\kappa & =\frac{\gamma^{F}}{1+u_{1}^{M}(t-1)-u_{2}^{M}(t-1)},
\end{aligned}
$$

where $\gamma^{\mathrm{F}}$ is the temporal discount rate of feelings $\left(0<\gamma^{\mathrm{F}}<1\right), N^{E}$ is the number of emotional inputs (the information of 48 adjectives $\cdot 2$ gestures $\cdot 2$ smiles), $w^{\mathrm{E}_{i, b, c, d}}$ is the weight between the $(b, c, d)$ emotion and the $i$ th feeling $\left(0 \leq w_{i, b, c, d}^{E} \leq 1\right), N^{\mathrm{F}}$ is the number of feelings $\left(N^{\mathrm{F}}=8\right), w^{F_{i, j}}$ is the stimulation or suppression coefficient from the $j$-th feeling to the $i$-th feeling $\left(0 \leq w^{\mathrm{F}}, b \leq 1\right)$, and $u^{M}{ }_{m}(t)$ is the value of the $m$-th mood. We use positive mood $(m=1)$ and negative mood $(m=2)$. The hyperbolic tangent is used to regulate the values of feelings. Mood is defined as the long-term state updated by a change in feelings, and governs changes in feelings. Feeling is defined as a short-term state updated by a change in emotion. The state of the $m$-th mood is updated by the sum of feelings:

$$
u_{m}^{M}(t)=\tanh \left[\gamma^{M} u_{m}^{M}(t-1)+\left(1-\gamma^{M}\right) \sum_{i=1}^{N^{F}} w_{m, i}^{M} u_{i}^{F}(t)\right],
$$

where $\gamma^{\mathrm{M}}$ is the discount rate and $w^{M}{ }_{m, i}$ is the stimulation or suppression coefficient from the $i$-th feeling to the $m$-th $\operatorname{mood}\left(0 \leq w^{M}{ }_{m, i} \leq 1\right)$.

\subsection{Recurrent Simple Spike Response Model}

There are several causes for human emotional changes. For example, emotions arise based on either internal or external changes [33].

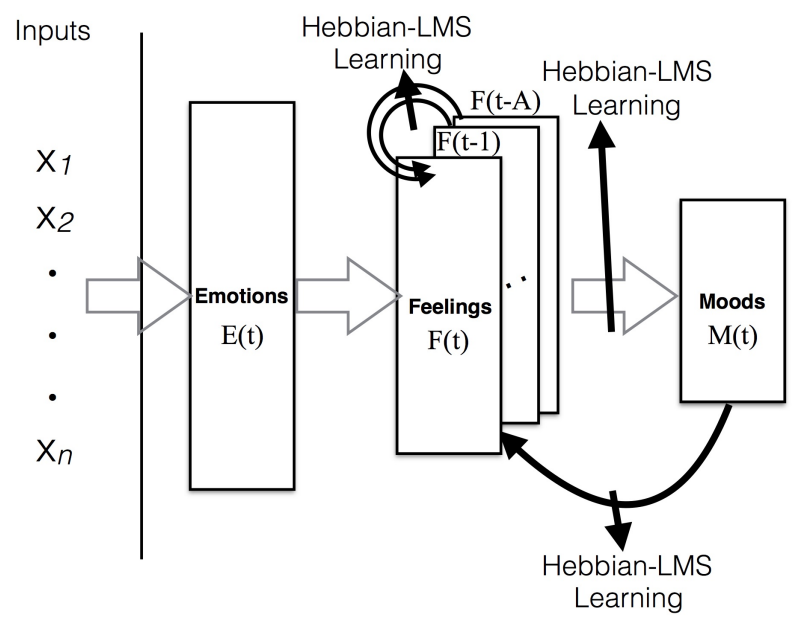

Fig. 6: The learning structure of the recurrent simple spike response model 
Human emotions are stored in memory, where the memory affects human current emotions, appraisals and personality [23]. Thus, we apply recurrent structured spiking neural network to realize the memory (Fig. 6).

In order to reduce the computational cost for the smart device application, a modified simple spike response model is applied in this paper. In this model, the internal state (membrane potential) $h_{i}(t)$ of the $i$-th neuron at the discrete time $t$ is described by the following equation:

$$
h_{i}(t)=\tanh \left(h_{i}^{s y n}(t)+h_{i}^{e x t}(t)+h_{i}^{r e f}(t)\right)
$$

From Eq.(5) we can see that the internal state of the neuron depends on three components:

- $h^{\text {syn }}{ }_{i}(t)$ includes the weighted pulse outputs from the other neurons and the reduced value of the internal state in the previous timestep. In this paper, we use a recurrent feeling layer (Figs.6, 7). Therefore, we have modified the equation for calculating $h^{\text {syn }}(t)$ as follows:

$$
h_{i}^{s y n}(t)=\sum_{a=1}^{A}\left(\gamma^{s y n}(t-a) \cdot h_{i}(t-a)+\sum_{j=1, i \neq j}^{N} w_{j, i}(t-a) \cdot h_{j}^{P S P}(t-a)\right),
$$

where $\gamma^{\text {syn }}(t-a)$ is the temporal discount rate of $h_{i} ; w_{j, i}(t-a)$ is a weight from the $j$-th neuron to the $i$-th neuron at the discrete time $t-a ; h_{j}{ }^{P S P}(t-a)$ is the Post Synaptic Potential (PSP) approximately transmitted from the $j$-th neuron at the discrete time $t-a ; N$ is the number of neurons; and $A$ indicates the recurrence. In this paper, we use recurrence in the feeling layer, where $A=2$ as shown in Fig. 7. In the emotion layer and in the mood layer, $A=1$.

- $h^{\text {ext }}(t)$ is the input to the $i$-th neuron from the environment.

- $h^{r e f}{ }_{i}(t)$ is used for representing the refractoriness of the neuron. This means that after the neuron fired, its internal state value is decreased using the refractoriness component, in order to avoid the continuous firing of the neuron within a short period of time.

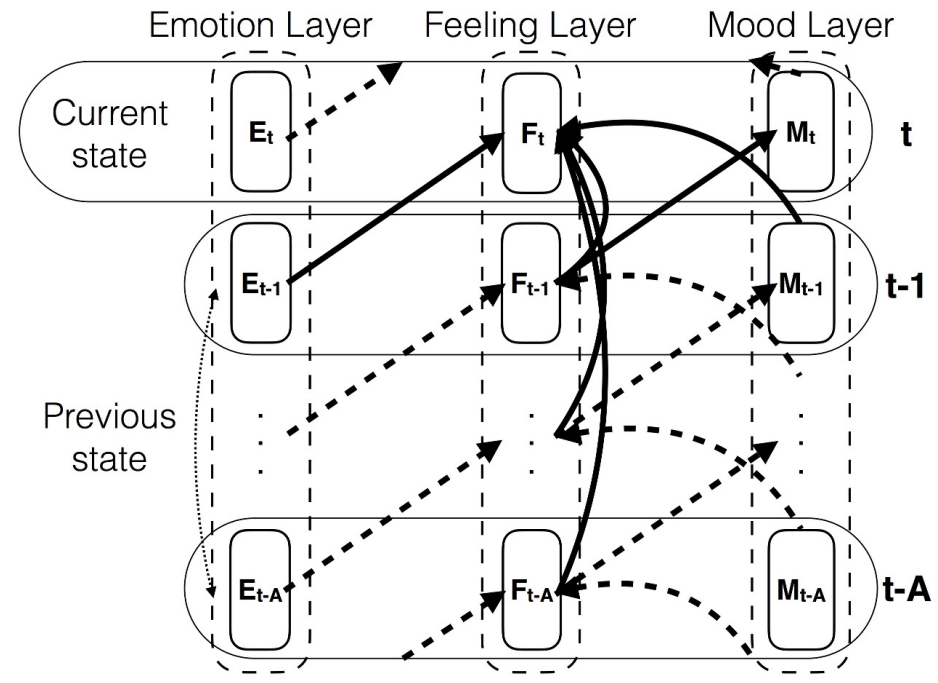

Fig. 7: The structure of the recurrent state

To avoid the bursting of neuronal fires, the hyperbolic tangent function is used in Eq. (5). When the internal state of the $i$-th neuron reaches a predefined threshold level, a pulse is outputted as follows: 


$$
p_{i}(t)= \begin{cases}1 & \text { if } h_{i}(t) \geq \theta \\ 0 & \text { otherwise }\end{cases}
$$

where $\theta$ is a threshold for firing. In case of firing, $R$ is subtracted from the $h^{\text {ref }}(t)$ value of neuron $i$ :

$$
h_{i}^{r e f}(t)= \begin{cases}\gamma^{r e f} \cdot h_{i}^{r e f}(t-1)-R & \text { if } p_{i}(t-1)=1 \\ \gamma^{r e f} \cdot h_{i}^{r e f}(t-1) & \text { otherwise }\end{cases}
$$

where $R>0, \gamma^{\text {ref }}$ is a discount rate of $h^{r e f}$, and $0 \leq \gamma^{\text {ref }} \leq 1$. The Post Synaptic Potential is the response of the postsynaptic neuron to a presynaptic action potential [15]. This is illustrated in Fig. 8, where the $j$-th neuron is the presynaptic neuron (sender) and the $i$-th neuron is the postsynaptic neuron (receiver). The presynaptic spike output is transmitted to the connected neuron through the weight connection as shown in Fig. 8:

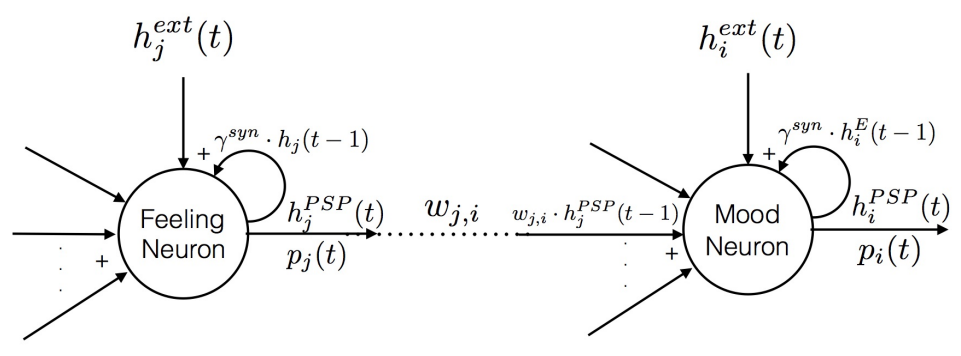

$\begin{array}{ll}\text { (a) } j \text {-th feeling } & \text { (b) } i \text {-th mood }\end{array}$

Fig. 8: The structure of the simple spike response model

$$
h_{i}^{P S P}(t)= \begin{cases}1 & \text { if } p_{i}(t)=1, \\ \gamma^{P S P} \cdot h_{i}^{P S P}(t-1) & \text { otherwise }\end{cases}
$$

where $\gamma^{P S P}$ is a discount rate of $h_{i}^{P S P}$ and $0<\gamma^{P S P}<1$. The PSP is excitatory if the weight parameter, $w_{j, i}$ is positive.

\subsection{Spiking Neural Network with Hebbian-LMS Learning}

In this paper, we propose a recurrent simple spike response model based emotional model, and we apply "Hebbian- LMS" learning to improve the robot's personal emotional system [43]. Here, we explain the learning algorithm divided into three parts: Emotion, Feeling, and Mood layer (Fig. 6).

\subsubsection{Emotion Layer}

$h_{g}{ }^{E}(t)$ is the perceptual information from smart device in the emotional layer part. $h_{g}{ }^{E-s y n}(t)$ has no weight pulse outputs from the other neurons, because the emotion neurons are not interconnected. Hence:

$$
h_{g}^{E-s y n}(t)=\gamma^{E-s y n}(t-1) \cdot h_{g}^{E}(t-1)
$$

where the $E$ index means emotion neuron based on the sensory information from the smart device. Emotion neurons are connected to feeling neurons (Fig. 9). The $W^{E}$ parameters are predefined to form the personality of the robot as presented in Section 4.5.1. 


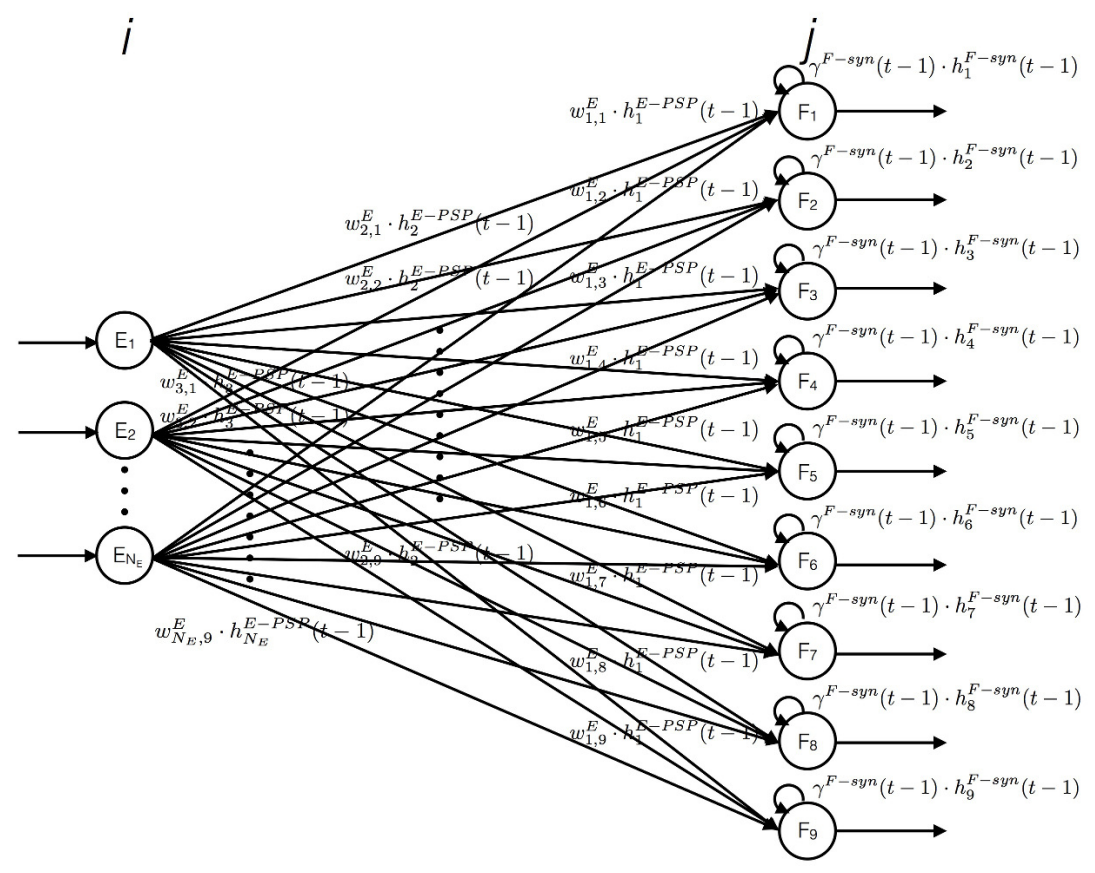

Fig. 9: The structure of spiking neural network: Emotion layer $(i)$ to Feeling layer $(j)$

\subsubsection{Feeling Layer}

The external input of the neurons in the feeling layer is calculated from the presynaptic spike output of emotion neurons and mood neurons as depicted in Fig. 10:

$$
h_{j}^{F-e x t}(t)=\sum_{g=1, g \neq j}^{N_{E}} w_{g, j}^{E} \cdot h_{g}^{E-P S P}(t-1)+\sum_{k=1, k \neq j}^{N_{M}} w_{k, j}^{M F}(t-1) \cdot h_{k}^{M-P S P}(t-1)
$$

where the $F$ index means feeling neuron; $N_{E}$ is the number of emotion neurons; $w^{E}{ }_{g, j}$ is the weight between the $g$-th emotion neuron and the $j$-th feeling neuron (Fig. 9); $N_{M}$ is the number of mood neurons; and $w^{M F}$ is the weight between the $k$-th mood neuron and the $j$-th feeling neuron (Fig. 11). The learning equation is shown in Section 4.5.4, where $W^{F}$ is the connection weight between the previous feeling and the current feeling (Fig. 12). The calculation of $h^{F-s y n}(t)$ for the interconnected feeling neurons is described in Eq. (6) (Feeling layer: $A=2$ ). 


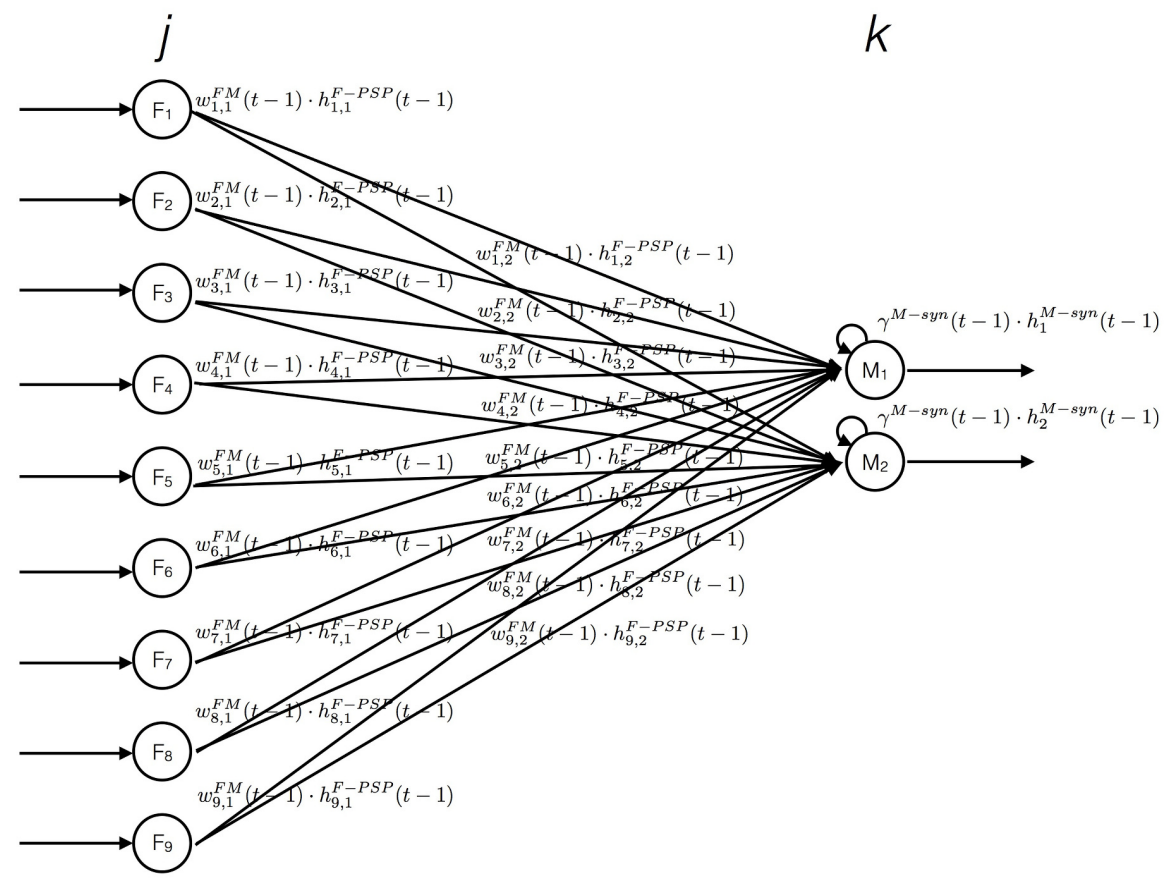

Fig. 10: The structure of spiking neural network: Feeling layer (j) to Mood layer (k)

\subsubsection{Mood Layer}

The external input of the neurons in the mood layer is calculated from the presynaptic spike output of the feeling neurons. The basic structure of neurons is shown in Fig. 10:

$$
h_{k}^{M-e x t}(t)=\sum_{k=1}^{N_{F}} w_{j, k}^{F M}(t-1) \cdot h_{j}^{F-P S P}(t-1)
$$

where the $M$ index means mood neuron; $N_{F}$ is the number of feeling neurons; and $w^{F M}{ }_{j, k}$ is the weight between the $j$-th feeling neuron and the $k$-th mood neuron. In the calculation of $h^{M-s y n}{ }_{k}(t)$, no weighted pulse outputs from the other neurons are considered, because the mood neurons are not interconnected, thus in $h^{M-s y n}{ }_{k}(t)$ only the previous mood state value is used:

$$
h_{k}^{M-s y n}(t)=\gamma^{M-s y n}(t-1) \cdot h_{k}^{M}(t-1)
$$

where the $k$ index means mood layer. The mood layer has two mood factors $\left(N_{M}=2\right)$ : positive $(+)$ and negative $(-)$. In [5], we did not consider the feedback structure from mood to feeling layer. Here, we consider this feedback. The equation is shown in Section 4.5.3. 


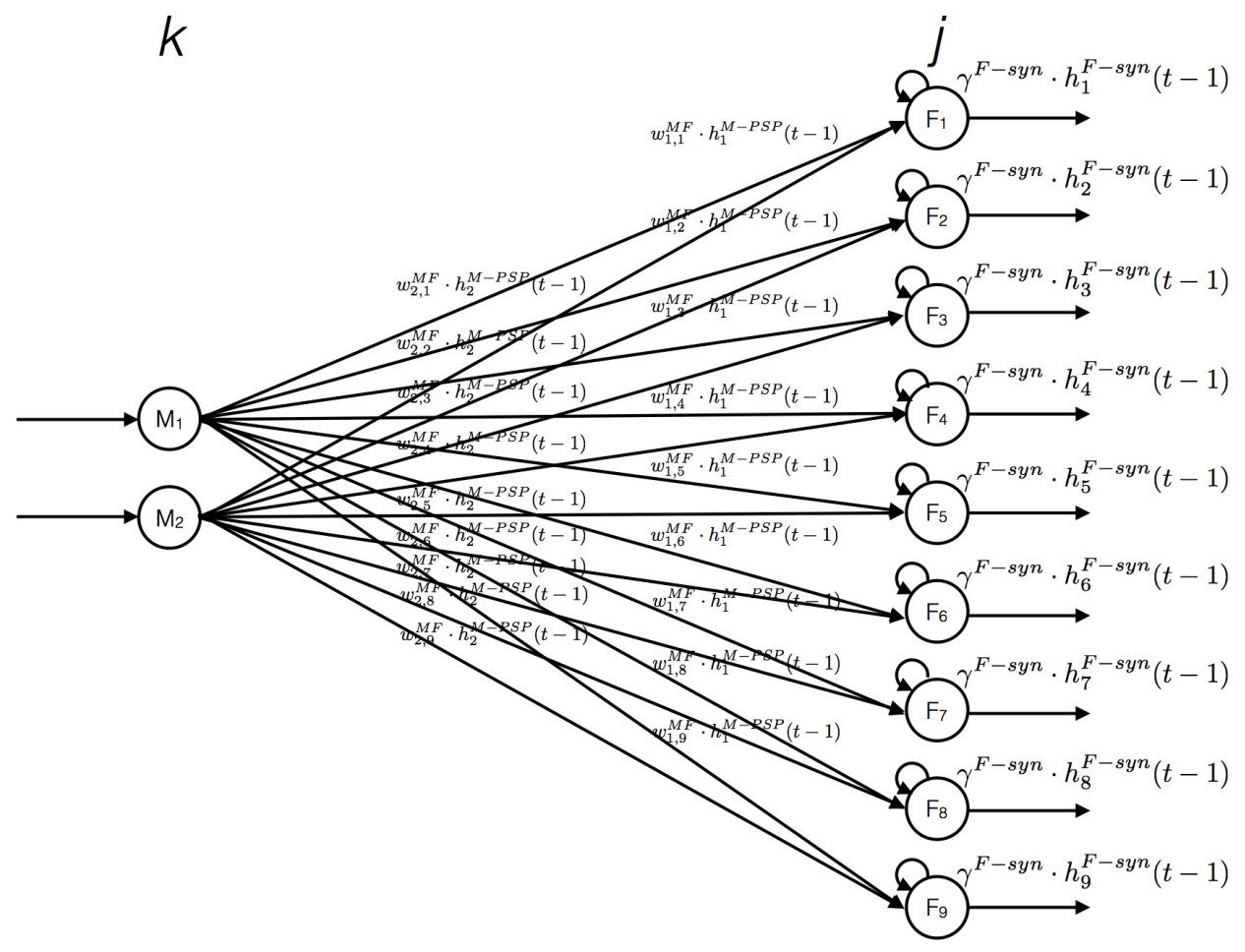

Fig. 11: The structure of spiking neural network: Mood layer (k) to Feeling layer (j)

\subsection{Weight Learning by Hebbian-LMS}

The basic equation of "The Hebbian-LMS Learning Algorithm" is as follows [43]:

$$
\begin{aligned}
W_{k+1} & =W_{k}+2 \mu e_{k} X_{k} \\
e_{k} & =S G M\left(X_{k}^{T} W_{k}\right)-\gamma X_{k}^{T} W_{k}=S G M\left((S U M)_{k}\right)-\gamma(S U M)_{k}
\end{aligned}
$$

where $\mu$ controls stability and convergence speed parameter; $X_{k}$ is the input pattern; $(S U M)_{k}$ is the sum of inputs; $\gamma$ is the slope parameter to control the stable equilibrium point; $k$ is the time step. For our spiking neural network based emotional model we have modified the equation of learning weight as follows:

$$
\begin{aligned}
W(t) & =W(t-1)+Z \circ X_{\text {input }}(t-1) \\
Z & =\xi^{\text {wgt }}\left(\tanh \left(S U M_{\text {input }}\right)-\gamma^{w g t} \cdot\left(S U M_{\text {input }}\right)\right)
\end{aligned}
$$

where $\xi^{\text {wgt }}$ is a coefficient to control stability and convergence speed; $\gamma^{\text {wgt }}$ is a parameter to control the stable equilibrium point; $\circ$ is "Hadamard Product". In this paper, $S U M_{\text {input }}$ consists of two parts as the input parameters: PostSynaptic Potential (PSP) and the self-connection values. The feeling layer is related to three weights for learning: $W^{F M}$, $W^{M F}$ and $W^{F}$. We show these weight parameters in Sections 4.5.2, 4.5.3 and 4.5.4.

\subsubsection{Learning Weight: $W^{E}$}

We use predefined weight parameters of $W^{E}$ as presented in Tables A-3, A-4, A-5 and A- 6 . It determines the robot's personal emotion state. The weight parameters are used as illustrated in Fig. 9. In order to realize robot emotional model, we define basic emotional input information such as feeling parameter ( $w^{\left.E_{\text {gfeeling. }}\right)}$ ) from Section 4.2 , battery $\left(w^{E}{ }_{\text {gbattery }, j}\right)$, distance $\left(w^{E}{ }_{\text {gdistance }, j}\right)$, and gender $\left(w_{\text {ggender }, j}^{E}\right)$ information by using smart device (Table 2). Each weight parameter has predefined parameters for robot personality. These parameters are combined as follows in Eq. (16).

$$
w_{g, j}^{E}=\frac{w_{g_{\text {feeling }, j}}^{E}+w_{g_{\text {battery }}, j}^{E}+w_{g_{\text {distance }, j}}^{E}+w_{g_{g e n d e r}, j}^{E}}{4}
$$




\subsubsection{Learning Weight: $W^{F M}$}

$W^{F M}$ is the learning weight for connections between feelings and moods as shown in Fig.10. The basic learning equation is as follows:

$$
W_{j, k}^{F M}(t)=W_{j, k}^{F M}(t-1)+Z_{k} \circ X_{\text {input }}^{F M}(t-1)
$$

The extended version of this equation is shown in Eq. (A-1) in Appendix, and $Z_{k}$ is shown in Eq. (18). The initial weight parameter $\left(W^{F M}\right)$ for robot personality is predefined by using Table 5 .

$$
\begin{aligned}
Z_{k} & =\xi^{w g t} \cdot \tanh \left(\left[w_{1, k}^{F M}(t-1), \cdots, w_{9, k}^{F M}(t-1), \gamma^{M-s y n}(t-1)\right]\right. \\
\cdot & {\left.\left[h_{1, k}^{F-P S P}(t-1), \cdots, h_{9, k}^{F-P S P}(t-1), h_{k}^{M-s y n}(t-1)\right]^{T}\right) } \\
& -\xi^{w g t} \cdot \gamma^{w g t} \cdot\left[w_{1, k}^{F M}(t-1), w_{2, k}^{F M}(t-1), \cdots, w_{9, k}^{F M}(t-1), \gamma^{M-s y n}(t-1)\right] \\
\cdot & {\left[h_{1, k}^{F-P S P}(t-1), h_{2, k}^{F-P S P}(t-1), \cdots, h_{k}^{M-s y n}(t-1)\right]^{T} }
\end{aligned}
$$

\subsubsection{Learning Weight: $W^{M F}$}

$W^{M F}$ is the learning weight for connections between moods and feelings as illustrated in Fig. 11. The basic learning equation is as follows:

$$
W_{k, j}^{M F}(t)=W_{k, j}^{M F}(t-1)+Z_{j} \circ X_{\text {input }}^{M F}(t-1)
$$

The extended version of this equation is shown in Eq. (A-2) in Appendix and $Z_{j}$ is shown in Eq. (20). The initial weight parameter $\left(W^{M F}\right)$ is initialized by " 0 ".

$$
\begin{gathered}
Z_{j}=\xi^{w g t} \cdot \tanh \left(\left[w_{1, j}^{M F}(t-1), w_{2, j}^{M F}(t-1), \gamma^{F-s y n}(t-1)\right]\right. \\
\left.\cdot\left[h_{1}^{M-P S P}(t-1), h_{2}^{M-P S P}(t-1), h_{j}^{F-s y n}(t-1)\right]^{T}\right) \\
-\xi^{w g t} \cdot \gamma^{w g t} \cdot\left[w_{1, j}^{M F}(t-1), w_{2, j}^{M F}(t-1), \gamma^{F-s y n}(t-1)\right] \\
\cdot\left[h_{1}^{M-P S P}(t-1), h_{2}^{M-P S P}(t-1), h_{j}^{F-s y n}(t-1)\right]^{T}
\end{gathered}
$$

Table 5: The initial weight parameter: $W^{F M}$

\begin{tabular}{|l|c|c|}
\hline$W^{F M}$ & Positive & Negative \\
\hline Neutral & 0.2 & 0.2 \\
\hline Happy & 0.7 & 0.0 \\
\hline Surprise & 0.7 & 0.0 \\
\hline Angry & 0.0 & 0.7 \\
\hline Disgust & 0.0 & 0.7 \\
\hline Sad & 0.0 & 0.7 \\
\hline Frightened & 0.0 & 0.7 \\
\hline Fearful & 0.0 & 0.7 \\
\hline Thrilling & 0.7 & 0.0 \\
\hline
\end{tabular}

\subsubsection{Learning Weight: $W^{F}$}

$W^{F}$ is the learning weight for connections between feelings including previous feelings as well, as shown in Fig. 12. The basic learning equation is as follows:

$$
W_{i, j}^{F}(t)=\sum_{a=1}^{A}\left(W_{i, j}^{F}(t-a)+Z_{j}(t-a) \circ X_{i n p u t}^{F}(t-a)\right)
$$


where $A$ is the recurrent steps. The extended version of this equation is shown in Eq. (A-3) in Appendix and $Z_{i, j}$ $(t-a)$ is shown in Eq. (22). The weight parameters $\left(W^{F}\right)$ are initialized by " 0 ". In Eq. (A-3), $h^{\varphi}{ }_{i, j}(t-a)$ is defined by Eq. (23), $w^{\varphi}(t-a)$ is defined by Eq. (24), and $z_{i, j}(t-a)$ is defined by Eq.(25).

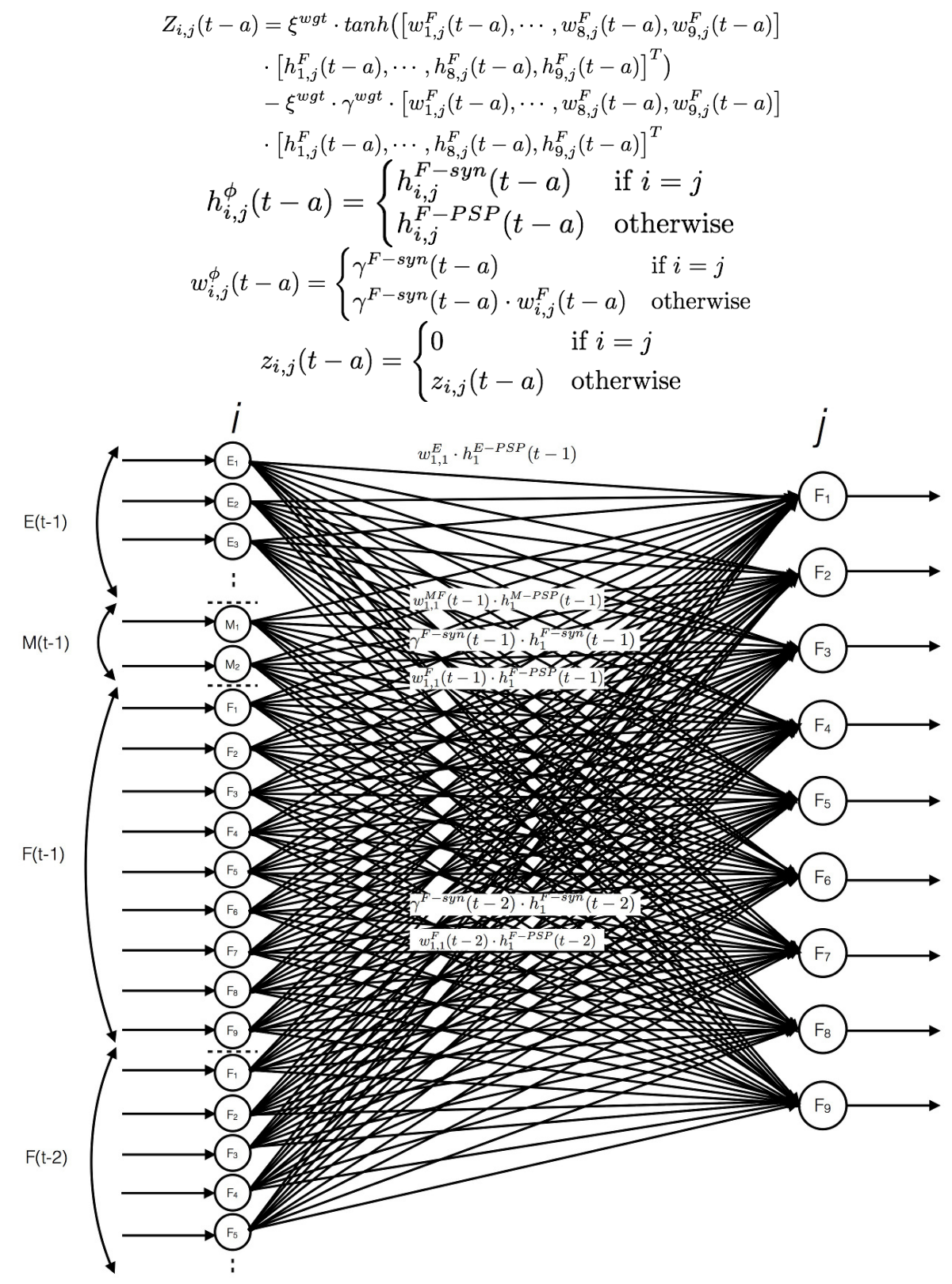

Fig. 12: The structure of spiking neural network: Other layers $(i)$ to Feelings $(j)$

\subsection{EXPERIMENTAL RESULTS}

The experimental results consist of three parts. First, we show the estimation of human emotional state based on our proposed human empathy model. Then, we apply a previously proposed emotional model for our robot partner. Finally, we apply our proposed recurrent spiking neural network based emotional model for our robot partner and compare it with the previous model. In order to indicate difference of emotional expression according to gender, we used two different experiment conditions based on gender difference. 


\subsection{Estimation of Human Emotional State}

In the experiment, we use "iPhonoid-C" as the robot partner. We also applied this definition of robot character in our previous paper [6]. This experiment is performed in a one-to-one conversation environment between the human and the robot partner. Human interaction condition has eleven steps as shown in Table 6, where $(t)$ is the time step from one to eleven depending on the condition of human. In each step $(t)$ there are two calculation parts. The first part is human emotion estimation, which takes 100 iterations for learning. The second part is the robot's emotion calculation, which takes 200 iterations for learning.

The robot used perception based emotional model for the estimation of human empathy as discussed in Section 4.2 The estimation of emotional state is changed based on each step $(t)$. For example, the robot could estimate angry situation based on the utterance information in step 7. In step 10, the feeling state is sad because the human leaves the room without the robot. Fig. 13 shows the estimation of feeling results and Fig. 14 shows the estimation of mood results. Fig. 15 depicts the winner information of emotional state for each result. The winner information of feeling is used to calculate the robot emotional model as human emotional state information.

Table 6: Summary of Human State

\begin{tabular}{|c|c|c|c|c|c|c|}
\hline \multirow{2}{*}{$(t)$} & \multicolumn{3}{|c|}{ Human emotional factor } & \multicolumn{3}{c|}{ Robot emotional factor } \\
\cline { 2 - 7 } & Gesture & Smiling & Adjective & Battery & Distance & Gender \\
\hline 1 & None & None & None & Low & Far away & Female $\cdot$ Male \\
\hline 2 & None & None & None & Full & Close & Female $\cdot$ Male \\
\hline 3 & None & Smiling & None & Full & Close & Female $\cdot$ Male \\
\hline 4 & Up \& Down & None & None & Full & Close & Female $\cdot$ Male \\
\hline 5 & None & Smiling & Happy & Full & Close & Female $\cdot$ Male \\
\hline 6 & None & None & Surprise & Full & Close & Female $\cdot$ Male \\
\hline 7 & None & None & Angry & Full & Close & Female $\cdot$ Male \\
\hline 8 & None & None & Surprise & Full & Close & Female $\cdot$ Male \\
\hline 9 & None & Smiling & Happy & Full & Close & Female $\cdot$ Male \\
\hline 10 & Left \& Right & Smiling & None & Full & Close & Female $\cdot$ Male \\
\hline 11 & None & None & None & Full & Far away & Female $\cdot$ Male \\
\hline
\end{tabular}

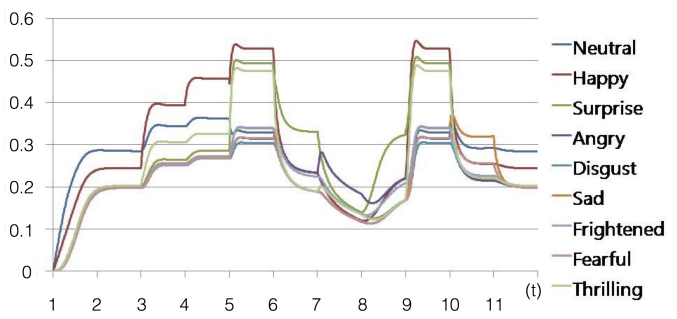

Fig. 13: The results of feeling to estimate human state

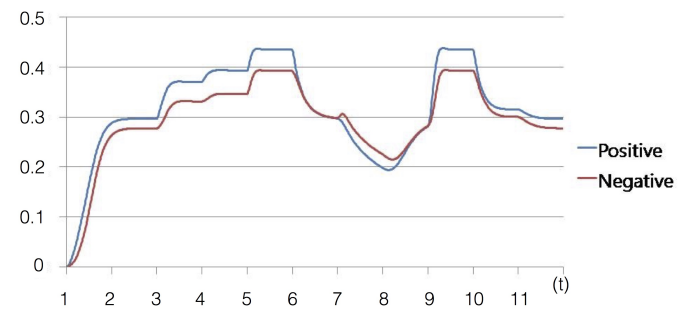

Fig. 14: The results of mood to estimate human state

\subsection{The Perception-based Emotional Model for the Robot}

In this part, we apply the perception-based emotional model for the robot partner [46]. The experimental conditions are the same as in Table 6. The calculation is repeated 200 times for each step information from human robot interaction in order to illustrate the calculation for changing in the emotional state. To compare the difference of emotional results, we performed two experiments with different genders to interact with the robot.

Our previous paper [46] used predefined emotional parameters for $W^{F}$ and $W^{F M}$ as presented in Tables 7 and 8. The equations in Section 4.2 are used in this emotional model. The same input parameters are used as in Section 4.5.1 The robot has a good impression to female based on the predefined gender parameters. Therefore, the robot has a sad feeling before the female is leaving because it is lonely when it is left alone in the room (Figs. 
$16,18,20)$. The robot's emotional state is similar to the human's emotional state. However, the robot is a little angry to male because the male left without carrying away the robot (Figs. 17, 19, 21).

This emotional model uses predefined parameters in advance. Thus, in this case, the robot's emotional expression is far from an autonomous emotional system. Consequently, we proposed a spiking neural network based emotional model [5].

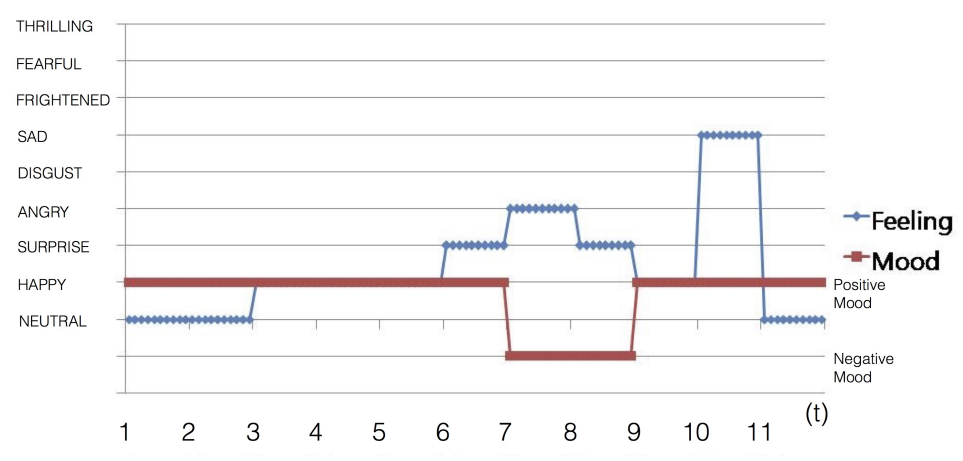

Fig. 15: The results of human emotional state: feeling and mood

Table 7: Coefficients between feelings $\left(W^{F}\right)$

\begin{tabular}{|l|c|c|c|c|c|c|c|c|c|}
\hline$W^{F}$ & Neutral & Happy & Surprise & Angry & Disgust & Sad & Frightened & Fearful & Thrilling \\
\hline Neutral & 0.0 & 0.3 & 0.1 & 0.1 & 0.1 & 0.1 & 0.1 & 0.1 & 0.3 \\
\hline Happy & 0.1 & 0.0 & 0.3 & 0.1 & 0.1 & 0.1 & 0.1 & 0.1 & 0.3 \\
\hline Surprise & 0.1 & 0.3 & 0.0 & 0.3 & 0.1 & 0.1 & 0.1 & 0.1 & 0.1 \\
\hline Angry & 0.1 & 0.1 & 0.3 & 0.0 & 0.3 & 0.1 & 0.1 & 0.1 & 0.1 \\
\hline Disgust & 0.1 & 0.1 & 0.1 & 0.3 & 0.0 & 0.3 & 0.1 & 0.1 & 0.1 \\
\hline Sad & 0.1 & 0.1 & 0.1 & 0.1 & 0.3 & 0.0 & 0.3 & 0.1 & 0.1 \\
\hline Frightened & 0.1 & 0.1 & 0.1 & 0.1 & 0.1 & 0.3 & 0.0 & 0.3 & 0.1 \\
\hline Fearful & 0.1 & 0.1 & 0.1 & 0.1 & 0.1 & 0.1 & 0.3 & 0.0 & 0.3 \\
\hline Thrilling & 0.1 & 0.3 & 0.1 & 0.1 & 0.1 & 0.1 & 0.1 & 0.3 & 0.0 \\
\hline
\end{tabular}

Table 8: Coefficients between feelings and moods $\left(W^{F M}\right)$

\begin{tabular}{|l|c|c|c|c|c|c|c|c|c|}
\hline & Neutral & Happy & Surprise & Angry & Disgust & Sad & Frightened & Fearful & Thrilling \\
\hline Positive & 0.2 & 0.7 & 0.2 & 0.1 & 0.1 & 0.1 & 0.1 & 0.1 & 0.1 \\
\hline Negative & 0.1 & 0.1 & 0.2 & 0.7 & 0.2 & 0.1 & 0.1 & 0.1 & 0.1 \\
\hline
\end{tabular}

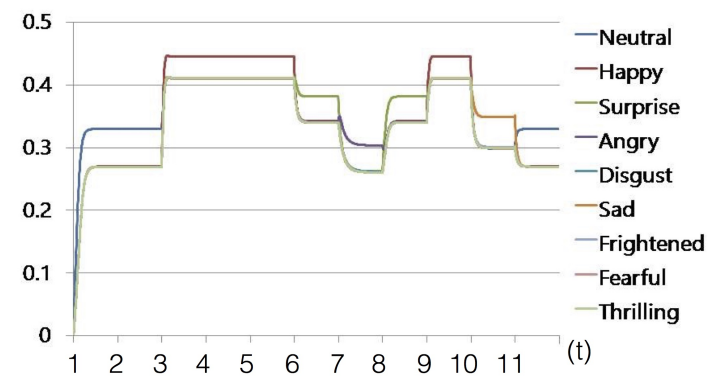

Fig. 16: The results of feeling: Female

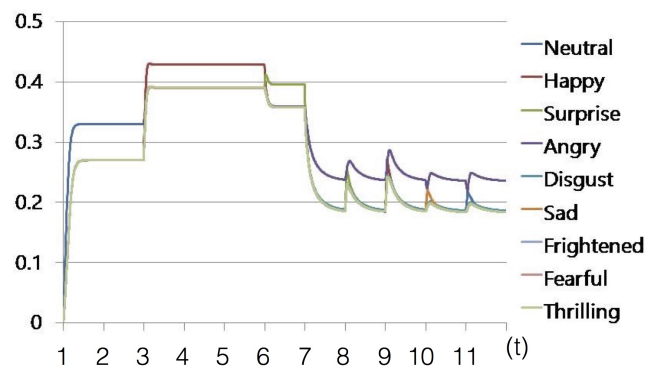

Fig. 17: The results of feeling: Male 


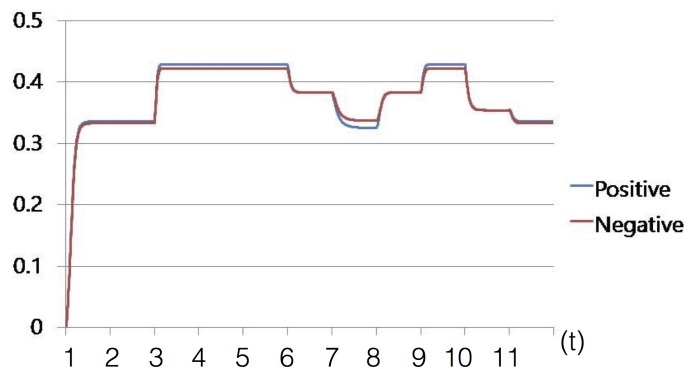

Fig. 18: The results of mood: Female

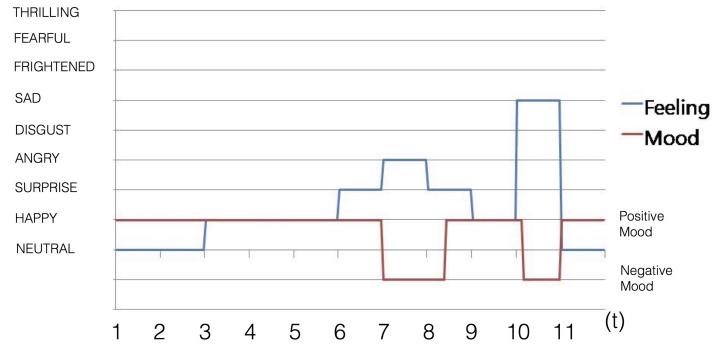

Fig. 20: The results of emotional states: Female

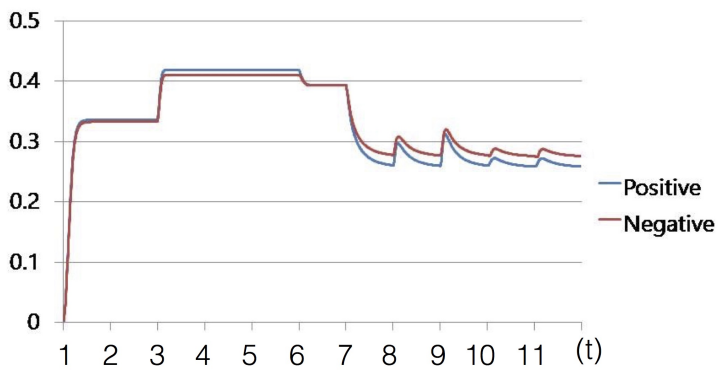

Fig. 19: The results of mood: Male

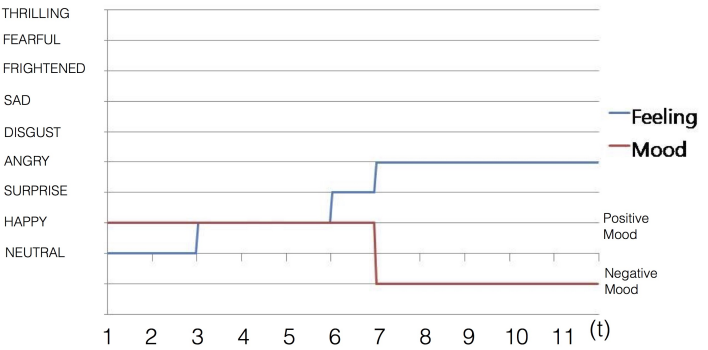

Fig. 21: The results of emotional states: Male

\subsection{The Proposed Emotional Model for Robot}

In our proposed emotional model, the robot uses four emotion factors as shown in Section 4.5.1 The parameters of the SNN are presented in Table 9. These parameters seemed optimal after a trial and error process. The calculation is repeated 200 times per each input information from human robot interaction in order to illustrate the calculation for changing in the emotional state.

The human feeling information results used in the robot's emotional model to define the robot's emotional state are based on Section 4.2 To compare the difference of emotional results, we performed two experiments with different genders to interact with the robot. The estimation of human emotional condition is changed based on the eleven steps, as shown in Table 6.

Human emotional state has the same results as depicted in Figs. 13, 14, and 15. The robot's emotional inputs are illustrated in Fig. 22 based on the human empathy emotional state. This is a projection from the human emotional condition from Fig. 15. Accordingly, the robot's feelings are changing as shown in Figs. 23 and 24. The robot's mood are also changing as shown in Figs. 25 and 26. The results of feeling and mood are changing automatically by unsupervised learning. The results of the robot partner's emotional state are shown in Figs. 27 and 28. The result shows the difference between female and male, because the robot has different parameters which is due to its preference of female over male (Table A-6). Thus, the robot has happy feeling and positive mood from step $(t)=9$ as presented in Fig. 27. So, the robot's emotional state is automatically changing by using human emotional condition and automating the fine tuning of the parameters.

The final learning values of $W^{F M}, W^{M F}$ and $W^{F}$ are shown in Tables 10,11 , and 12 . The changing of the weight values during 200 steps is illustrated in Figs. 29, 30, and 31. Fig. 29 presents the changing of the weights $W^{F M}$ between all feelings and positive mood. Fig. 30 shows the cha nging of weights $W^{M F}$ from positive mood to all feelings. The learning time of $W^{M F}$ takes longer, because the mood is placed in the last layer. Thus, the $W^{M F}$ values are changed slower. The $W^{F}$ values are changing dynamically based on the relation of emotions, moods, and previous feelings (Fig. 31). As a result, all weights could increase and decrease during the learning process, showing an advantage of the Hebbian-LMS learning. 


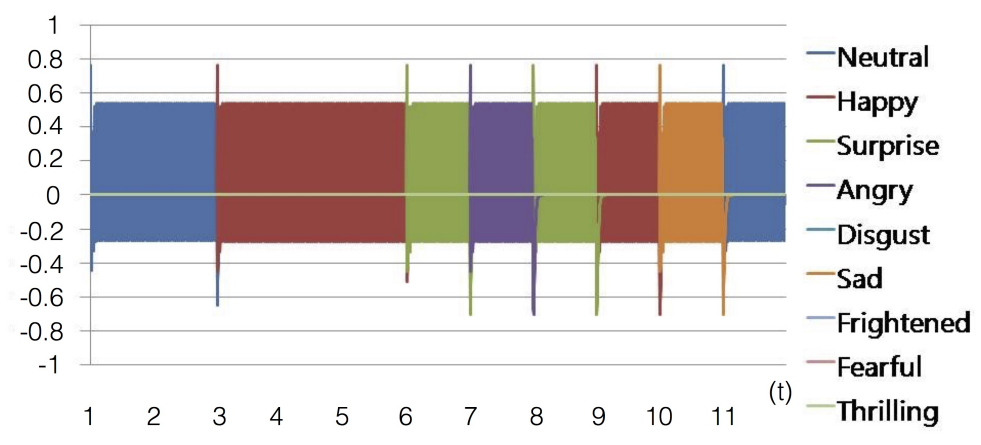

Fig. 22: The results of robot's emotional estimation: Output of Emotion Layer

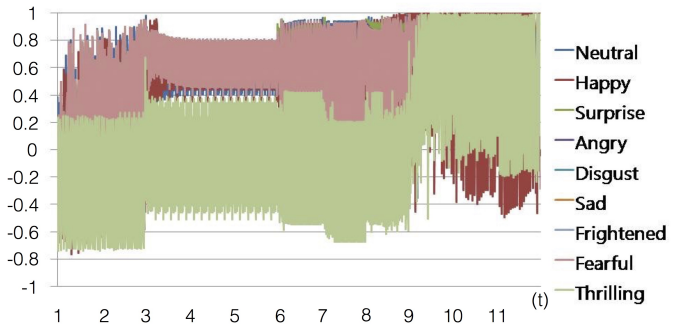

Fig. 23: The results of feeling: Female

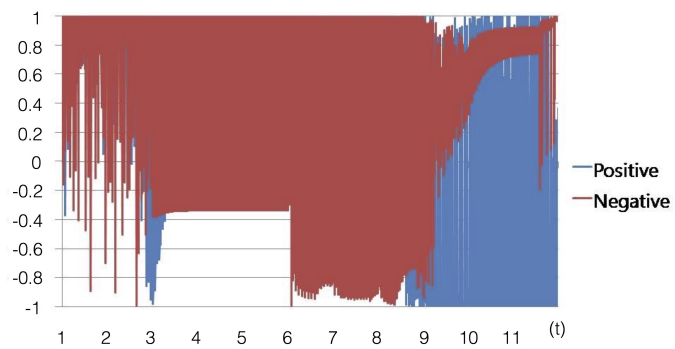

Fig. 25: The results of mood: Female

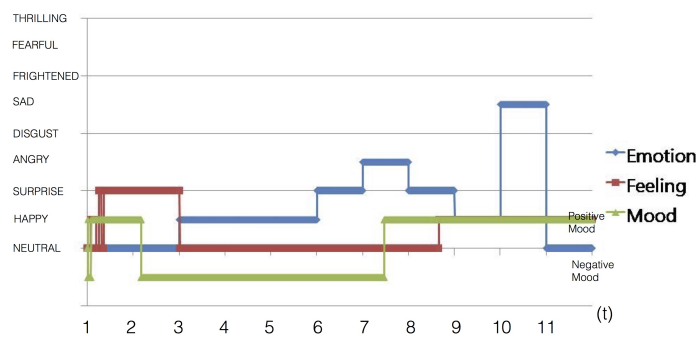

Fig. 27: The results of emotional states: Female

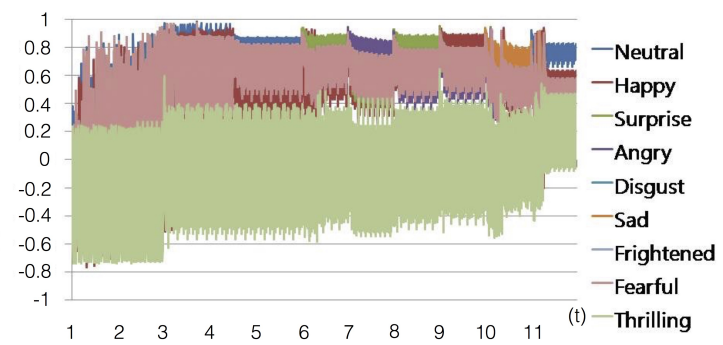

Fig. 24: The results of feeling: Male

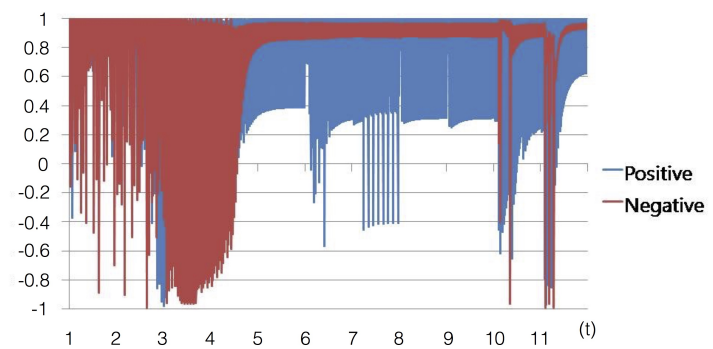

Fig. 26: The results of mood: Male

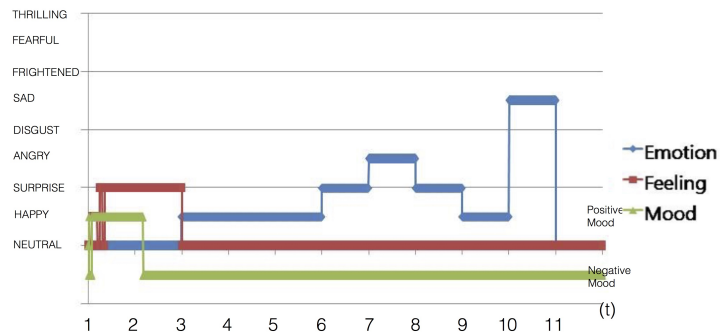

Fig. 28: The results of emotional states: Male 
Table 9: Parameter setting for emotional model by Spiking neural network

\begin{tabular}{|c|c|c|c|c|c|}
\hline$N_{E}$ & $\gamma^{E-s y n}(t-a)$ & $\gamma^{E-P S P}$ & $\gamma^{E-r e f}$ & $R_{E}$ & $\theta_{E}$ \\
\hline 9 & 0.55 & 0.9 & 0.7 & 1 & 0.2 \\
\hline$N_{F}$ & $\gamma^{F-s y n}(t-a)$ & $\gamma^{F-P S P}(t-a)$ & $\gamma^{F-r e f}$ & $R_{F}$ & $\theta_{F}$ \\
\hline 9 & $\begin{array}{c}0.35(a=1), \\
0.14(a=2)\end{array}$ & $\begin{array}{l}0.8(a=1), \\
0.6(a=2), \\
0.4(a=3)\end{array}$ & 0.7 & 1 & 0.2 \\
\hline$N_{M}$ & $\gamma^{M-s y n}(t-a)$ & $\gamma^{M-P S P}$ & $\gamma^{M-r e f}$ & $R_{M}$ & $\theta_{M}$ \\
\hline 2 & 0.8 & 0.85 & 0.55 & 1 & 0.2 \\
\hline & $\bar{A}$ & \multicolumn{2}{|c|}{$\gamma^{\text {wgt }}$} & \multicolumn{2}{|c|}{$\xi^{w g t}$} \\
\hline & 2 & \multicolumn{2}{|c|}{0.7} & \multicolumn{2}{|c|}{0.3} \\
\hline
\end{tabular}

Table 10: Final values of weights after learning (Female): $W^{F M}$

\begin{tabular}{|l|c|c|}
\hline$W^{F M}$ & Positive & Negative \\
\hline Neutral & 0.2 & 0.2 \\
\hline Happy & 0.7 & 0.0 \\
\hline Surprise & 0.7 & 0.0 \\
\hline Angry & 0.0 & 0.7 \\
\hline Disgust & 0.0 & 0.7 \\
\hline Sad & 0.0 & 0.7 \\
\hline Frightened & 0.0 & 0.7 \\
\hline Fearful & 0.0 & 0.7 \\
\hline Thrilling & 0.7 & 0.0 \\
\hline
\end{tabular}

Table 11: Final values of weights after learning (Female): $W^{M F}$

\begin{tabular}{|l|c|c|c|c|c|c|c|c|c|}
\hline$W^{M F}$ & Neutral & Happy & Surprise & Angry & Disgust & Sad & Frightened & Fearful & Thrilling \\
\hline Positive & 0.2 & 0.7 & 0.2 & 0.1 & 0.1 & 0.1 & 0.1 & 0.1 & 0.1 \\
\hline Negative & 0.1 & 0.1 & 0.2 & 0.7 & 0.2 & 0.1 & 0.1 & 0.1 & 0.1 \\
\hline
\end{tabular}

Table 12: Final values of weights after learning (Female): $W^{F}$

\begin{tabular}{|l|c|c|c|c|c|c|c|c|c|}
\hline$W^{F}$ & Neutral & Happy & Surprise & Angry & Disgust & Sad & Frightened & Fearful & Thrilling \\
\hline Neutral & 0.00 & 0.40 & 0.42 & 0.41 & 0.41 & 0.41 & 0.41 & 0.41 & 0.39 \\
\hline Happy & 0.30 & 0.00 & 0.31 & 0.31 & 0.33 & 0.32 & 0.32 & 0.32 & 0.47 \\
\hline Surprise & 0.33 & 0.34 & 0.00 & 0.33 & 0.35 & 0.34 & 0.34 & 0.34 & 0.42 \\
\hline Angry & 0.33 & 0.34 & 0.33 & 0.00 & 0.35 & 0.35 & 0.35 & 0.35 & 0.43 \\
\hline Disgust & 0.38 & 0.41 & 0.38 & 0.38 & 0.00 & 0.37 & 0.37 & 0.37 & 0.38 \\
\hline Sad & 0.39 & 0.41 & 0.38 & 0.38 & 0.37 & 0.00 & 0.37 & 0.37 & 0.38 \\
\hline Frightened & 0.39 & 0.41 & 0.38 & 0.38 & 0.37 & 0.37 & 0.00 & 0.37 & 0.38 \\
\hline Fearful & 0.39 & 0.41 & 0.38 & 0.38 & 0.37 & 0.37 & 0.37 & 0.00 & 0.38 \\
\hline Thrilling & 0.32 & 0.35 & 0.32 & 0.33 & 0.34 & 0.34 & 0.34 & 0.34 & 0.00 \\
\hline
\end{tabular}




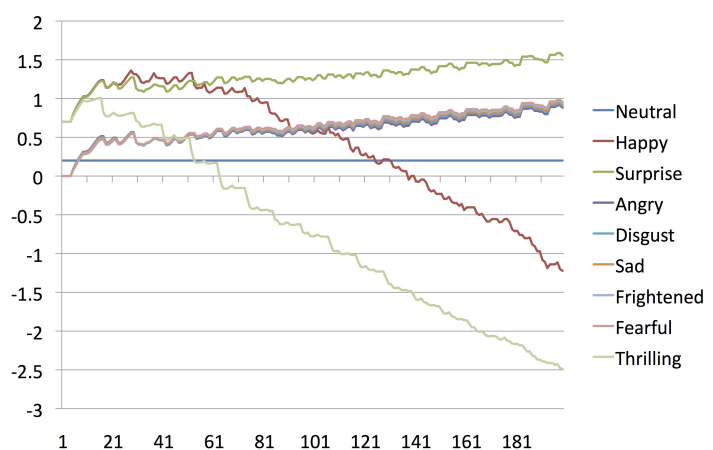

Fig. 29: The changing of weight values (Female): $\mathrm{W}^{F M}$ Fig.

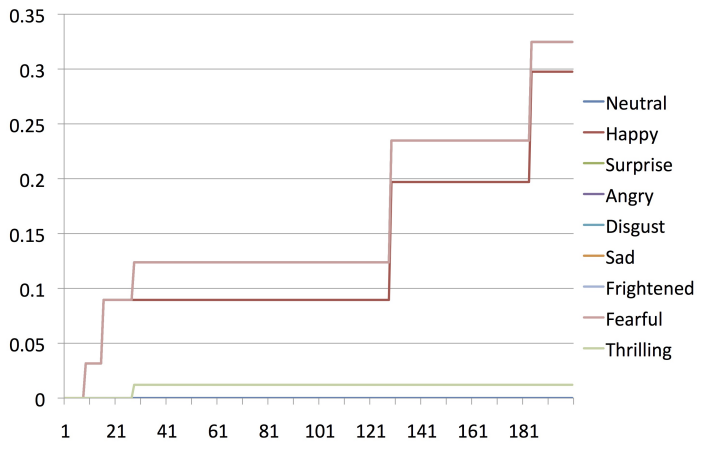

Fig. 30: The changing of weight values (Female):

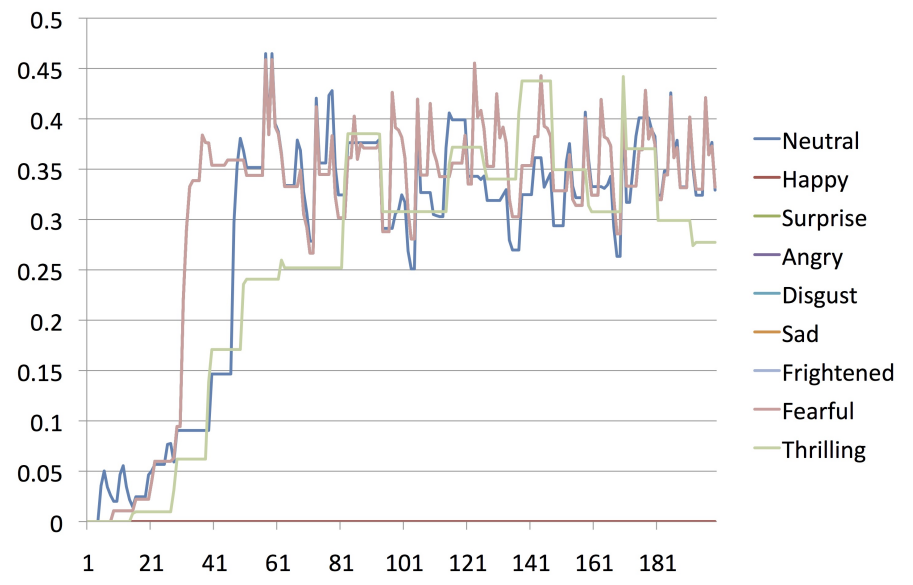

Fig. 30: The changing of weight values (Female): $\mathrm{W}^{F}$

\subsection{CONCLUSION}

In this paper, we proposed an emotional empathy structure based on human empathy and robot emotion (Fig. 5). First, we used emotional estimation for human empathy. This result is used as an emotional input for the estimation of the robot's emotional state. Thus, the robot's emotional state has empathy structure with human emotional state.

The previous robot emotional model was not automatic, it has predefined parameter settings [46]. To improve our emotional model, we proposed a recurrent simple spike response model based on the "Hebbian-LMS" learning for the robot's emotional model. The advantage of the learning method is that it does not require parameter tuning by human, so it automatizes the fine tuning of parameters.

We used the original Hebbian learning rule in our previous approach. Sometimes, the weights reached their maximum values after some learning cycles. The weight could not decrease in that case. However, in this paper, we could avoid this problem by the "Hebbian-LMS" learning. The weight values can increase and decrease as well. Here, emotional model has complex dynamics. Therefore, it is difficult to advance the design manually. Thus, the robot partner should generate enriched emotional expression by its own through the introduction of unsupervised learning.

For future work, we will consider using the recurrent spiking neural network model with Hebbian-LMS learning for Natural Language Processing (NLP) to realize more natural conversation. Finally, we intend to try the emotional empathy model for multi-robot environment. 


\section{APPENDIX}

\section{A.1 Acronyms}

Table A-1: Acronyms

\begin{tabular}{|c|l|}
\hline Acronyms & \multicolumn{1}{|c|}{ Full name } \\
\hline LMS & Least Mean Square \\
\hline CFUS & Conversation Flow Utterance System \\
\hline SBUS & Sentence Building Utterance System \\
\hline TDUS & Time Dependent Utterance System \\
\hline LMA & Laban Movement Analysis \\
\hline PSP & Post Synaptic Potential \\
\hline syn & synaptic \\
\hline ext & external \\
\hline ref & refractoriness \\
\hline
\end{tabular}

\section{A.2 Notations}

Table A-2: Notations

\begin{tabular}{|c|c|}
\hline Notations & Meanings \\
\hline$E$ & emotion \\
\hline$F$ & feeling \\
\hline$M$ & mood \\
\hline$S_{b}$ & $b$-th adjective word \\
\hline$G_{c}$ & $c$-th gesture information from human \\
\hline$D_{d}$ & $d$-th smiling information from human \\
\hline$u^{E}$ & emotion input \\
\hline$u^{F}$ & feeling input \\
\hline$u^{M}$ & mood input \\
\hline$w^{E}$ & the learning weight parameter of emotion \\
\hline$w^{F}$ & the learning weight parameter of feeling \\
\hline$w^{M}$ & the learning weight parameter of mood \\
\hline$w^{F M}$ & $\begin{array}{l}\text { the learning weight parameter between feeling to mood } \\
\text { in spiking neural network }\end{array}$ \\
\hline$w^{M F}$ & $\begin{array}{l}\text { the learning weight parameter between mood to feeling } \\
\text { in spiking neural network }\end{array}$ \\
\hline$N^{E}$ & the number of emotions \\
\hline$N^{F}$ & the number of feelings \\
\hline$N^{M}$ & the number of moods \\
\hline$\gamma^{F}$ & the discount rate of feelings \\
\hline$\gamma^{M}$ & the discount rate of moods \\
\hline$h_{i}$ & $i$-th membrane potential \\
\hline$h_{i}^{\text {syn }}$ & $i$-th synaptic pulse output \\
\hline$h_{i}{ }^{e x t}$ & $i$-th external information from environent \\
\hline$h_{i}^{\text {ref }}$ & $i$-th refractoriness of the neuron \\
\hline$h_{i}{ }^{P S P}$ & $i$-th presynaptic spike output \\
\hline$p_{i}$ & pulse output \\
\hline$\gamma^{\text {syn }}$ & the discount rate of $h$ \\
\hline$\gamma^{r e f}$ & the discount rate of $h^{r e f}$ \\
\hline
\end{tabular}




\begin{tabular}{|c|l|}
\hline$\gamma^{P S P}$ & the discount rate of $h^{P S P}$ \\
\hline$\theta$ & Threshold for firing \\
\hline$\xi^{\text {wgt }}$ & the coefficient to control stability and convergence speed \\
\hline$\gamma^{\text {wgt }}$ & parameter to control the stable equilibrium point \\
\hline$X_{\text {input }}$ & the input pattern \\
\hline$(S U M)_{k}$ & sum of inputs \\
\hline
\end{tabular}

\section{A.3 Equations}

Eqs. (A-1), (A-2), and (A-3) are extended version of Eqs. (17), (19), and (21).

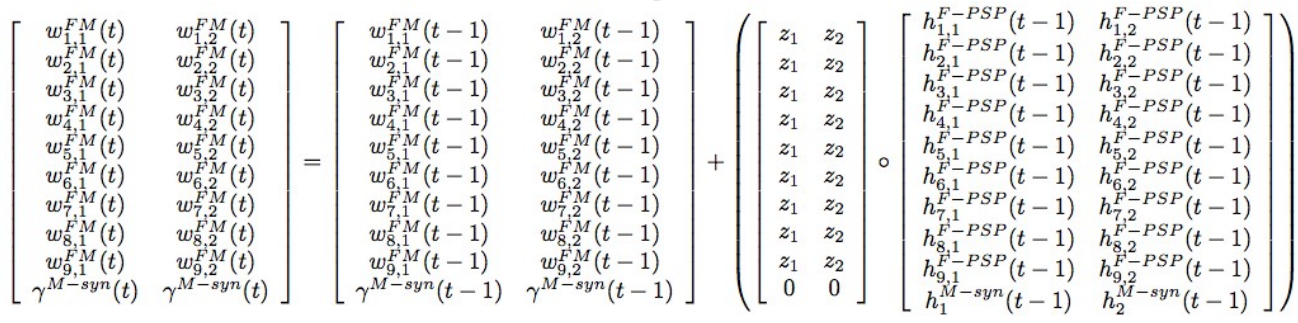

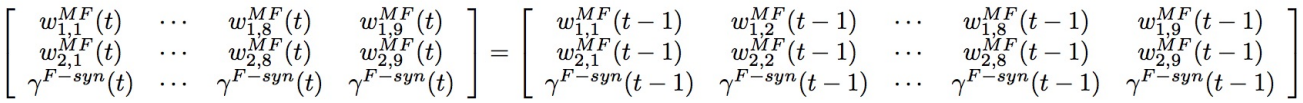

$$
\begin{aligned}
& +\left(\left[\begin{array}{ccc}
z_{1} & \cdots & z_{9} \\
z_{1} & \cdots & z_{9} \\
0 & \cdots & 0
\end{array}\right] \circ\left[\begin{array}{ccccc}
h_{1,1}^{M-P S P}(t-1) & h_{1,2}^{M-P S P}(t-1) & \cdots & h_{1,8}^{M-P S P}(t-1) & h_{1,9}^{M-P S P}(t-1) \\
h_{2,1}^{M-P S P}(t-1) & h_{2,2}^{M-P S P}(t-1) & \cdots & h_{2,8}^{M-P S P}(t-1) & h_{2,9}^{M-P S P}(t-1) \\
h_{1}^{F-s y n}(t-1) & h_{2}^{F-s y n}(t-1) & \cdots & h_{8}^{F-s y n}(t-1) & h_{9}^{F-S y n}(t-1)
\end{array}\right]\right)
\end{aligned}
$$

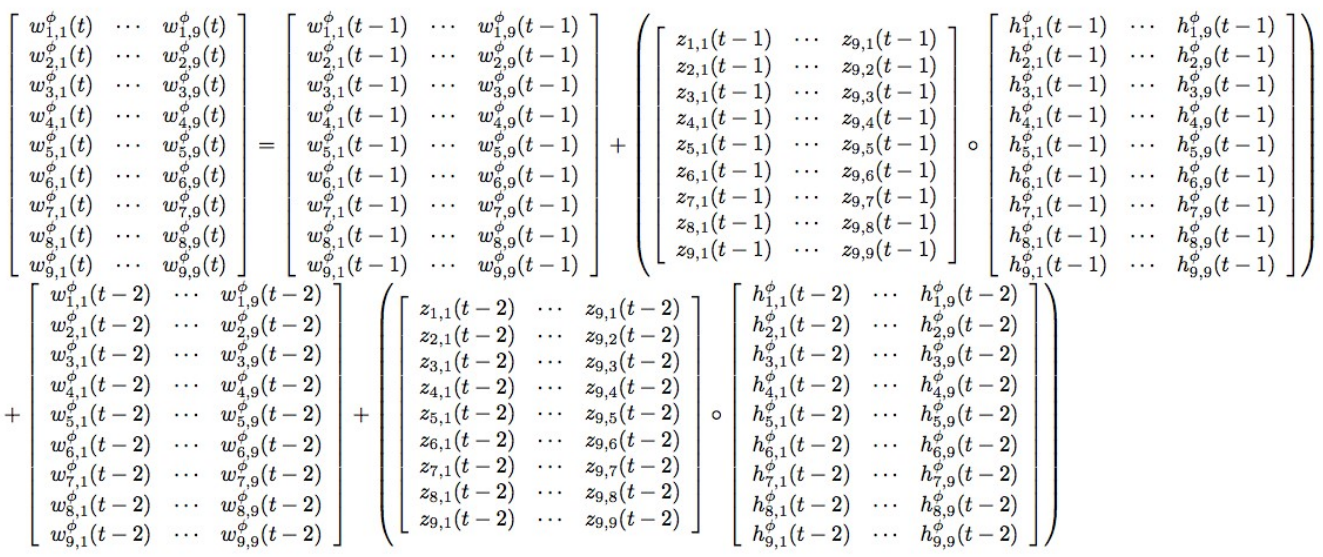

\section{A.4 Parameter Tables}

Tables A-3, A-4, A-5 and A-6 are coefficients in order to calculate the emotions based on the input information. Humans are learning about the emotional expression according to their experiences. However, robots need emotional parameter for each situation that is a reference of emotion (e.g., What is the robot's emotional state when robot has greeting situation.). Thus, we predefined robot partner's emotional parameter to calculate emotions for robot personality.

Table A-3: Coefficients of emotions $\left(w^{E}{ }_{\text {gfeeling.j. }}\right)$ : Human emotional state

\begin{tabular}{|c|c|c|c|c|c|c|c|c|c|}
\hline Feelings & Neutral & Happy & Surprise & Angry & Disgust & Sad & Frightened & Fearful & Thrilling \\
\hline Neutral & 1.0 & 0.0 & 0.0 & 0.0 & 0.0 & 0.0 & 0.0 & 0.0 & 0.0 \\
\hline Happy & 0.0 & 1.0 & 0.0 & 0.0 & 0.0 & 0.0 & 0.0 & 0.0 & 0.0 \\
\hline Surprise & 0.0 & 0.0 & 1.0 & 0.0 & 0.0 & 0.0 & 0.0 & 0.0 & 0.0 \\
\hline
\end{tabular}




\begin{tabular}{|c|l|l|l|l|l|l|l|l|l|}
\hline Angry & 0.0 & 0.0 & 0.0 & 1.0 & 0.0 & 0.0 & 0.0 & 0.0 & 0.0 \\
\hline Disgust & 0.0 & 0.0 & 0.0 & 0.0 & 0.0 & 1.0 & 0.0 & 0.0 & 0.0 \\
\hline Sad & 0.0 & 0.0 & 0.0 & 0.0 & 0.0 & 1.0 & 0.0 & 0.0 & 0.0 \\
\hline Frightened & 0.0 & 0.0 & 0.0 & 0.0 & 0.0 & 0.0 & 1.0 & 0.0 & 0.0 \\
\hline Fearful & 0.0 & 0.0 & 0.0 & 0.0 & 0.0 & 0.0 & 0.0 & 1.0 & 0.0 \\
\hline Thrilling & 0.0 & 0.0 & 0.0 & 0.0 & 0.0 & 0.0 & 0.0 & 0.0 & 1.0 \\
\hline
\end{tabular}

Table A-4: Coefficients of emotions ( $\mathrm{w}^{E}$ gbattery,$\left.j\right)$ : Battery state

\begin{tabular}{|c|c|c|c|c|c|c|c|c|c|}
\hline Battery & Neutral & Happy & Surprise & Angry & Disgust & Sad & Frightened & Fearful & Thrilling \\
\hline Low & 0.2 & 0.4 & 0.6 & 0.9 & 0.8 & 0.8 & 0.7 & 0.8 & 0.4 \\
\hline Full & 0.2 & 0.8 & 0.4 & 0.4 & 0.4 & 0.4 & 0.5 & 0.5 & 0.7 \\
\hline
\end{tabular}

Table A-5: Coefficients of emotions ( $\mathrm{w}^{E}$ gbattery, $)$ : Distance information

\begin{tabular}{|c|c|c|c|c|c|c|c|c|c|}
\hline Distance & Neutral & Happy & Surprise & Angry & Disgust & Sad & Frightened & Fearful & Thrilling \\
\hline Long & 0.2 & 0.4 & 0.6 & 0.9 & 0.8 & 0.8 & 0.7 & 0.8 & 0.4 \\
\hline Short & 0.2 & 0.8 & 0.4 & 0.4 & 0.4 & 0.4 & 0.5 & 0.5 & 0.7 \\
\hline
\end{tabular}

Table A-6: Coefficients of emotions $\left(\mathrm{w}^{E}{ }_{\text {gbattery }, j}\right)$ : Gender information

\begin{tabular}{|c|c|c|c|c|c|c|c|c|c|}
\hline Distance & Neutral & Happy & Surprise & Angry & Disgust & Sad & Frightened & Fearful & Thrilling \\
\hline Female & 0.2 & 0.9 & 0.5 & 0.3 & 0.3 & 0.2 & 0.2 & 0.2 & 0.6 \\
\hline Male & 0.2 & 0.4 & 0.8 & 0.8 & 0.7 & 0.5 & 0.6 & 0.7 & 0.4 \\
\hline
\end{tabular}

\section{REFERENCES}

[1] Apple Inc (2016) Core Location Framework Reference. https://developer.apple. com/library/prerelease/watchos/documentation/CoreLocation/Reference/ CoreLocation_Framework/index.html\#//apple_ref/doc/uid/TP40007123

[2] Apple Inc (2016) Core Motion Framework Reference. https://developer.apple.com/library/prerelease/watchos/documentation/CoreMotion/Reference/CoreMoti on_Reference/index.html\#//apple_ref/doc/uid/TP40009686

[3] Apple Inc (2016) UIKit Framework Reference. https://developer.apple.com/library/ prerelease/watchos/documentation/UIKit/Reference/UIKit_Framework/

[4] Bechara A, Damasio H, Damasio AR (2000) Emotion, Decision Making and the Orbitofrontal Cortex. Cere- bral cortex 10(3):295-307

[5] Botzheim J, Kubota N (2014) Spiking Neural Network based Emotional Model for Robot Partner. In: 2014 IEEE Symposium on Robotic Intelligence In Informationally Structured Space (RiiSS), IEEE, pp $1-6$

[6] Botzheim J, Woo J, Tay NNW, Kubota N, Yamaguchi T (2014) Gestural and facial communication with smart phone based robot partner using emotional model. In: World Automation Congress (WAC), 2014, IEEE, pp 644-649

[7] Breazeal C (2004) Function meets Style: Insights from Emotion Theory Applied to HRI. IEEE Transactions on Systems, Man, and Cybernetics, Part C: Applications and Reviews 34(2):187-194 
[8] Breazeal CL (2004) Designing sociable robots. MIT press

[9] Cambridge University Press 2016 (2016) Cambridge. http://dictionary.cambridge.org/

[10] Canli T, Desmond JE, Zhao Z, Gabrieli JD (2002) Sex differences in the neural basis of emotional memories. Proceedings of the National Academy of Sciences 99(16):10,789-10,794

[11] Moohebat M, Raj RG, Kareem SBA, Thorleuchter D (2015) Identifying ISI-indexed articles by their lexical usage: A text analysis approach, Journal of the Association for Information Science and Technology, 66(3), pp. 501-511. doi: 10.1002/asi.23194..

[12] Ekman P (1993) Facial expression and emotion. American psychologist 48(4):384

[13] Ekman P (2007) Emotions revealed: Recognizing faces and feelings to improve communication and emo- tional life. Macmillan

[14] Fischer AH, Rodriguez Mosquera PM, Van Vianen AE, Manstead AS (2004) Gender and culture differences in emotion. Emotion 4(1):87

[15] Gerstner W, Kistler WM (2002) Spiking neuron models: Single neurons, populations, plasticity. Cambridge university press

[16] Hebb DO (1949) The Organization of Behavior. Wiley and Sons, New York, USA

[17] Hume, D. (2012). Emotions and moods. Organizational behavior, 258-297.

[18] Yeow WL, Mahmud R, Raj RG (2014) An application of case-based reasoning with machine learning for forensic autopsy, Expert Systems with Applications, 41(7), pp. 3497-3505, ISSN 0957- 4174, http://dx.doi.org/10.1016/j.eswa.2013.10.054. (http://www.sciencedirect.com/science/article/pii/S0957 417413008713).

[19] Kipp M, Martin JC (2009) Gesture and emotion: Can basic gestural form features discriminate emotions? In: 2009 3rd International Conference on Affective Computing and Intelligent Interaction and Workshops, IEEE, pp 1-8

[20] Kmiec SM (2009) An analysis of sex differences in empathy and forgiveness

[21] Kozareva Z, Navarro B, Vá zquez S, Montoyo A (2007) Ua-zbsa: a headline emotion classification through web information. In: Proceedings of the 4th International Workshop on Semantic Evaluations, Association for Computational Linguistics, pp 334-337

[22] Levine DS (2007) Neural Network Modeling of Emotion. Physics of Life Reviews 4(1):37-63

[23] Levine LJ, Safer MA (2002) Sources of bias in memory for emotions. Current Directions in Psychological Science 11(5):169-173

[24] Lewis M (2000) The Emergence of Human Emotions. Handbook of emotions 2:265-280

[25] Lewis M, Haviland-Jones JM, Barrett LF (2010) Handbook of emotions. Guilford Press

[26] Lin J, Spraragen M, Zyda M (2012) Computational models of emotion and cognition. In: Advances in Cog- nitive Systems, Citeseer 
[27] Raj RG and Abdul-Kareem S (2011). A Pattern Based Approach for The Derivation Of Base Forms Of Verbs From Participles And Tenses For Flexible NLP. Malaysian Journal of Computer Science, 24(2), pp 63-72.

[28] Masuyama N, Islam MN, Seera M, Loo CK (2015) Application of emotion affected associative memory based on mood congruency effects for a humanoid. Neural Computing and Applications pp 1-16

[29] Nagamachi, M. (1995). Kansei engineering: a new ergonomic consumer-oriented technology for product development. International Journal of industrial ergonomics, 15(1), 3-11.

[30] Nicholson J, Takahashi K, Nakatsu R (2000) Emotion recognition in speech using neural networks. Neural computing and applications 9(4):290-296

[31] Plutchik, R. (2001). The Nature of Emotions Human emotions have deep evolutionary roots, a fact that may explain their complexity and provide tools for clinical practice. American Scientist, 89(4), 344-350.

[32] Riggio, R. E., Tucker, J., \& Coffaro, D. (1989). Social skills and empathy. Personality and individual differences, 10(1), 93-99.

[33] Salovey P, Mayer JD (1990) Emotional intelligence. Imagination, cognition and personality 9(3):185211

[34] Sayegh L, Anthony WP, Perrewe PL (2004) Managerial decision-making under crisis: The role of emotion in an intuitive decision process. Human Resource Management Review 14(2):179-199

[35] Raj RG and Balakrishnan V (2011). A Model For Determining The Degree Of Contradictions In Information. Malaysian Journal of Computer Science, 243, pp 160-167.

[36] SoftBank Robotics (2016) Pepper. https://www.ald.softbankrobotics.com/en

[37] Sperber D, Wilson D (1995) Relevance: Communication and Cognition. Blackwell Publishing

[38] Stiff, J. B., Dillard, J. P., Somera, L., Kim, H., \& Sleight, C. (1988). Empathy, communication, and prosocial behavior. Communications Monographs, 55(2), 198-213.

[39] Tanaka F, Isshiki K, Takahashi F, Uekusa M, Sei R, Hayashi K (2015) Pepper learns together with children: Development of an educational application. In: 2015 IEEE-RAS 15th International Conference on Humanoid Robots (Humanoids), IEEE, pp 270-275

[40] Terasawa Y, Fukushima H, Umeda S (2013) How does interoceptive awareness interact with the subjective experience of emotion? an fmri study. Human brain mapping 34(3):598-612

[41] Raj RG, Abdul-Kareem S, Information Dissemination And Storage For Tele-Text Based Conversational Systems' Learning, Malaysian Journal of Computer Science, 22(2), pp. 138-159.

[42] Van Boven L, White K, Huber M (2009) Immediacy bias in emotion perception: Current emotions seem more intense than previous emotions. Journal of Experimental Psychology: General 138(3):368

[43] Widrow B, Kim Y, Park D (2015) The Hebbian-LMS Learning Algorithm. IEEE Computational Intelligence Magazine 10(4):37-53

[44] Woo J, Shimazaki J, Matsuo Y, Masuta H, Lim Ho, Kubota N (2013) Communication based on Frankl's Psychology for Humanoid Robot Partners using Emotional Model. In: 2013 IEEE International Conference on Fuzzy Systems (FUZZ), IEEE, pp 1-8 
[45] Woo J, Botzheim J, Kubota N (2014) Facial and Gestural Expression Generation for Robot Partners. In: 2014 International Symposium on Micro-NanoMechatronics and Human Science (MHS), IEEE, pp 1-6

[46] Woo J, Botzheim J, Kubota N (2015) Verbal Conversation System for a Socially Embedded Robot Partner using Emotional Model. In: Proceedings of the 24th IEEE International Symposium on Robot and Human Interactive Communication, IEEE, pp 37-42

[47] Woo, J., Botzheim, J., \& Kubota, N. (2017). A modular cognitive model of socially embedded robot partners for information support. ROBOMECH Journal, 4(1), 10

[48] Woo, J., Botzheim, J., \& Kubota, N. (2017). System Integration for Cognitive Model of a Robot Partner. Intelligent Automation \& Soft Computing, pp 1-14

[49] Yorita A, Botzheim J, Kubota N (2013) Emotional Models for Multi-modal Communication of Robot Part- ners. In: 2013 IEEE International Symposium on Industrial Electronics (ISIE), IEEE, pp 1-6

[50] Zhe X, Boucouvalas A (2002) Text-to-emotion engine for real time internet communication. In: Proceedings of International Symposium on Communication Systems, Networks and DSPs, Citeseer, pp $164-168$

[51] Zuckerman, M., Klorman, R., Larrance, D. T., \& Spiegel, N. H. (1981). Facial, autonomic, and subjective components of emotion: The facial feedback hypothesis versus the externalizer-internalizer distinction. Journal of Personality and social Psychology, 41(5), 929. 